Classification

Physics Abstracts

79.20F $-32.80 \mathrm{M}-07.60 \mathrm{P}$

\title{
Microanalytical Imaging with Auger Electrons
}

\author{
Martin Prutton \\ Department of Physics, University of York, York YO1 5DD, UK
}

(Received February 6; accepted February 21, 1995)

\begin{abstract}
The detection of Auger electrons in a scanning electron microscope in order to form chemically specific images of the surfaces of solids is reviewed. The limits to sensitivity and spatial resolution are described and the current 'state of the art' is summarised. Some of the useful image processing tools are reviewed and then a small set of applications of the technique when used for the study of surface segregation, semiconducting devices and archaeology are described.
\end{abstract}

\section{Introduction}

This article is concerned with a type of electron microscopy which uses electron excited Auger electrons to form an image of a surface and the variations from place to place in it of the chemical composition. This might be called Surface micro-analytical imaging.

Spectroscopic imaging involves the acquisition of a great deal of data. Imagine, for example, the characterisation of the chemical composition of an area of a sample containing 6 chemical elements whose concentrations vary from place to place. At each position on the sample at which an observation is made the heights or areas of at least 6 peaks in a spectrum must be measured. The simplest approach might be to make a crude estimate of the peak heights by subtracting the measured background in the spectrum at an energy above or below the peak from the height of the spectrum at the peak. Thus, for 6 elements, a minimum of 12 measurements must be made. Consider that it is desired to build up a picture with 512 by 512 picture points (pixels). This means that at least 3,145,728 measurements are required to derive the 6 images - one for each chemical element. Clearly we are immediately in the realm of experiments controlled by digital computers. Should subsequent numerical processing of these images be required - as is inevitable if the images are to be quantified so as to have contrast representing the concentrations of the 6 elements at each pixel - then it is necessary to be careful about the precision with which these data are stored in the computer. Assume that 32 bit precision is necessary. This means that the data will require about 12.5 million bytes of memory capacity. Should the experiment being carried out require sets of such images to be collected - the objective may be to vary the sample temperature to change local concentrations, to study the progress of a chemical reaction or the fracture of a piece of a 
metallic alloy - then even the relatively cheap random access and hard disc storage facilities on modern computers soon become prohibitively expensive.

This technical difficulty can be reduced by using efficient schemes of data coding to store the information but there are many researchers who question the wisdom of acquiring images. This is largely because of the experimental time required to accumulate all of the information. As will be seen below there are always statistical criteria which determine the precision of each individual measurement and these criteria cause there to be minimum data acquisition times for each pixel. Depending upon the experiment being carried out and the techniques being used these minima can lead to quite long data acquisition times for each set of images. These times are frequently somewhere in the range between minutes to several hours. The question therefore arises as to whether it is more efficient to choose places in or on the sample by some criterion such as a characteristically different contrast in a rapidly measured scanning electron microscope (SEM) image. These places are then carefully observed using spectroscopy and the analysis performed at those places only. A slightly more detailed approach might be to scan a region of a sample along a line using spectroscopic imaging techniques and so reduce the data acquisition time from that required to collect a whole picture (a frame) to that required to collect a single line of that frame whilst ensuring that the line crosses the feature of interest. A criticism of these faster experimental methods is that it is very easy to miss features of special interest. One example will be shown below for which there is barely any contrast at all in the SEM image and yet the Auger image reveals a distinct layer structure in the sample. Another example which can occur is when unexpected regions or phases have occurred in the sample and they just happen to have identical contrast in whatever fast scanning technique is used to try to classify how many different kinds of regions are present in the sample. Often it is the occurrence of unexpected effects that are both interesting and valuable in science! Finally, it should be stressed that the human eye-brain combination is very powerful at spotting spatial correlations - it is often easy to spot a pattern of faint lines in a diffuse optical image for example - and by analysing at points or along lines the spatially correlated information about the sample is entirely or largely thrown away. Perhaps also we should bear in mind the cliche - 'a picture is worth a thousand words'!

This paper addresses some of the issues that arise in imaging microanalysis by placing particular emphasis upon scanning beam techniques. These are very widespread in use and are conveniently adaptable to computer controlled equipment. Further, Auger imaging of the surfaces of solids will be of central concern because this has been developed to an advanced level, is relevant to this issue of the journal commemorating Pierre Auger's work and because it illustrates the ways in which images can be combined to obtain more information than was readily apparent in the raw data. The methods which are described here can be extended to other microanalytical techniques and this extension has begun in some fields. It will be assumed that the reader is reasonably familiar with the principles and methods of surface spectroscopy that can be found in the book by Briggs and Seah [1] and the references therein. Finally, the methods of surface analysis require that experiments are carried out under ultra-high vacuum (UHV) conditions and so this is taken for granted throughout the material described here.

1.1 SCANNING AND DiRECT MiCROSCOPIES. - There are two modes of acquisition of analytical images - scanning and direct. The scanning modes utilise a focussed probe of particles or radiation which is scanned in an analogue or digital fashion across the sample under study. Some of the scattered particles or radiation intended for use in microanalysis are collected by a spectrometer/detector arrangement and the signal measured is either stored in the memory of a control computer or is used to modulate the brightness of the beam in a cathode ray tube display which is being scanned synchronously with the motion of the probe on the sample. This is the principle used in the well known SEM. The general arrangement is outlined in Figure 1a. The subject has 


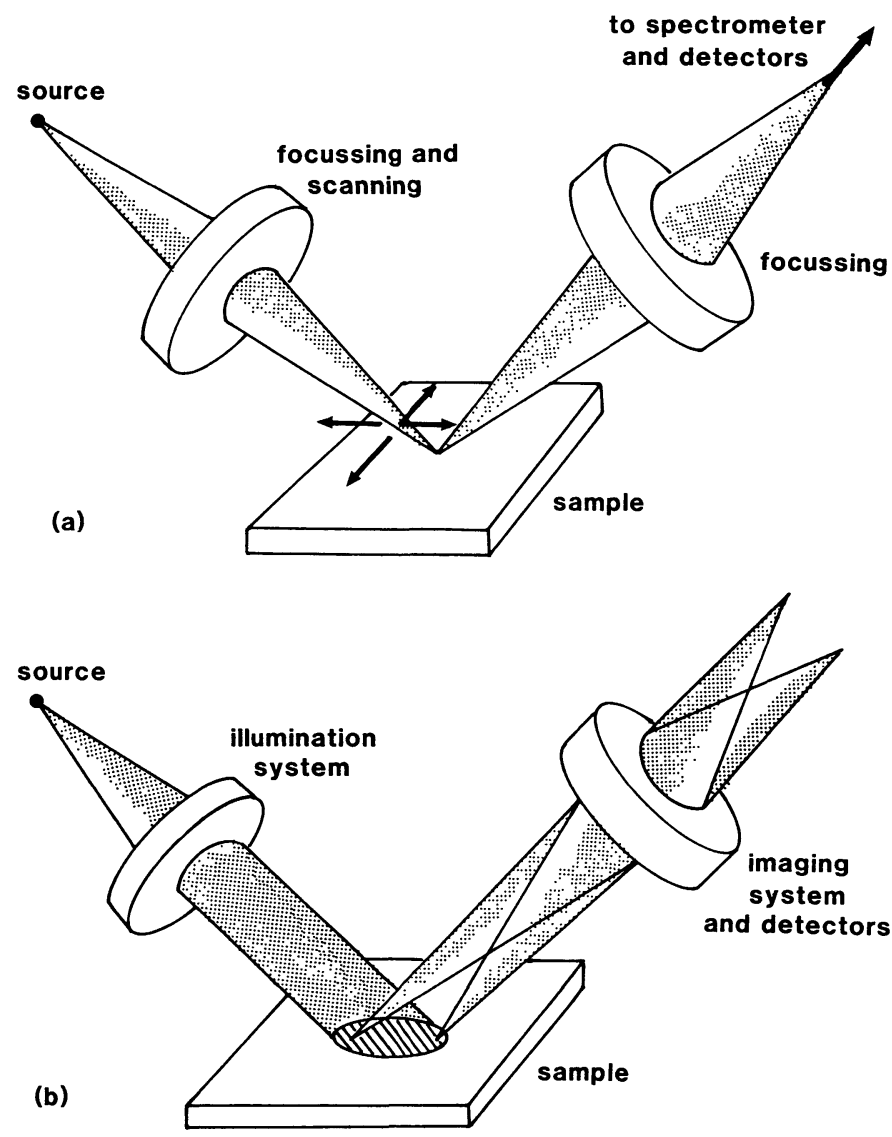

Fig. 1. - a) Scanning imaging - the incident beam may be focussed onto the sample and scanned across its surface as shown with spectrometer collecting electrons from the place which is illuminated. Alternatively, the surface may be flooded with electrons and a spectrometer has a small field of view scanned across the surface. b) Direct imaging - The surface is flooded with electrons and the spectrometer is designed to image the surface at the detector using electrons of a selected energy.

been extensively reviewed in a number of textbooks and research monographs. The SEM and the physics of the scattering of an incident electron beam into a variety of signals have been described by Reimer [2].

Direct imaging methods of microanalysis (Fig. 1b) are designed so that the sample is illuminated over a large area instead of the incident radiation being focussed into a fine probe. The spectrometer is of a type which images the emitting region of the sample onto a detector array. This kind of instrument has the substantial advantage that all regions in the area of interest are imaged simultaneously and so it is usually much faster than the scanned probe techniques. However, there is always a price to pay for improved performance and in these cases it is usually that the spectrometer is more complex (and thus expensive and more sophisticated in use) than those used in scanning instruments.

Attention will be focussed here upon scanning methods applied to Auger imaging. However, many of the data manipulation and interpretation techniques which will be described are applicable to the other kinds of images. It should be noted that a direct imaging spectrometer has 
been devised and used for photoelectron imaging by Turner et al. [3]. This instrument uses an imaging bandpass filter to select the energy at which an image is formed of an area illuminated by a photon beam. It cannot be used for incident electrons because the magnetic fields in the region of the imaging filter would distort the incident electron beam. However it could, perhaps, be used for imaging using photon excited Auger electrons. Direct imaging is also well established in Electron Energy Loss Spectroscopy and Imaging (EELSI) with the development of imaging filters as described by Krivanek [4].

1.2 Signal to Noise RAtios In IMAging. - Consider a beam of electrons with energy $E_{\mathrm{p}}$ and current $i_{\mathrm{b}}$ incident upon a solid sample. The atoms of the solid are ionised in a process with crosssection $\phi$ and emit Auger electrons into a spectrometer accepting a solid angle $\Omega$. The current of Auger electrons passing through the spectrometer will be $i_{\mathrm{A}}$, where:

$$
i_{\mathrm{A}}=i_{\mathrm{b}} \phi N r \lambda \Omega / 4 \pi
$$

In this equation $N$ is the number of atoms per unit volume generating Auger electrons of the correct energy to pass around the spectrometer and be detected. $r$ is the Auger backscattering factor. The quantity $\lambda$ is the inelastic mean free path (imfp) of the Auger electrons and a measure of the depth from which Auger electrons have escaped from the solid into the vacuum. This general form of the yield of Auger electrons was first proposed by Bishop and Riviere [5].

Equation (1) can be used to estimate the current of Auger electrons that may be detected in a particular situation. Consider for example a beam current of $10 \mathrm{nA}$ incident upon a monolayer of oxygen atoms adsorbed upon a silicon surface. Auger electrons with $505 \mathrm{eV}$ kinetic energy are emitted from the oxygen atoms which are present with a density of about $10^{15}$ atoms per square $\mathrm{cm}$. The cross-section is about $10^{-21} \mathrm{~cm}^{2}$. If, say, $1 \%$ of the electrons emitted enter the spectrometer and are detected and if the values of $r$ and $\lambda$ are taken as 1 and $10^{-9} \mathrm{~m}$ respectively then the current collected is about $10^{-15} \mathrm{~A}$. This is about 6000 electrons per second. If a measurement of this current is made by counting electrons for $\tau \mathrm{s}$ then $6000 \tau$ electrons are counted. Should repeated measurements of this count be taken then the set of measurements will have a standard deviation of $(6000 \tau)^{0.5}$ because of the Poisson statistics associated with the random arrival rate of electrons at the detector. Thus the signal to noise ratio will be about $6000 \tau /(6000 \tau)^{0.5}$ ie $(6000 \tau)^{0.5}$. Thus, for example, if a measurement is made for $17 \mathrm{~ms}$ then about 100 electrons will be detected and the signal to noise ratio will be $10: 1$.

This class of argument always indicates the basic limit to the sensitivity of an experiment in which particles are counted provided that it is the statistics of particle detection which sets the noise and not the performance of any associated detection electronics. For experiments which detect particles or photons other than Auger electrons all that is required is to replace equation (1) with the appropriate relationship between the exciting particles or radiation and the yield of detected particles or radiation. In scanning imaging experiments it is these signal to noise considerations that lead to the inconveniently long data acquisition times for a single image. Using the numbers given above a signal to noise ratio of $10: 1$ will require $17 \mathrm{~ms}$ per pixel per energy. If 6 energies are being acquired and a 512 by 512 pixel image is required then the total acquisition time must be at least $45 \mathrm{~min}$. Less time can be taken only by sacrificing signal to noise ratio or by reducing the pixel density. Even when making such sacrifices the improvement in acquisition time scales only with the square root of the quantity changed because the standard deviation of the data scales with the square root of the number of particles counted for each pixel. Alternatively, one might be free to increase the beam current $i_{\mathrm{b}}$. The difficulty here can be that a small beam size is required to obtain a good spatial resolution in the image and an increase in the beam current may cause an increase of the size of the spot into which the beam is focussed and so a loss of spatial resolution. Further, 

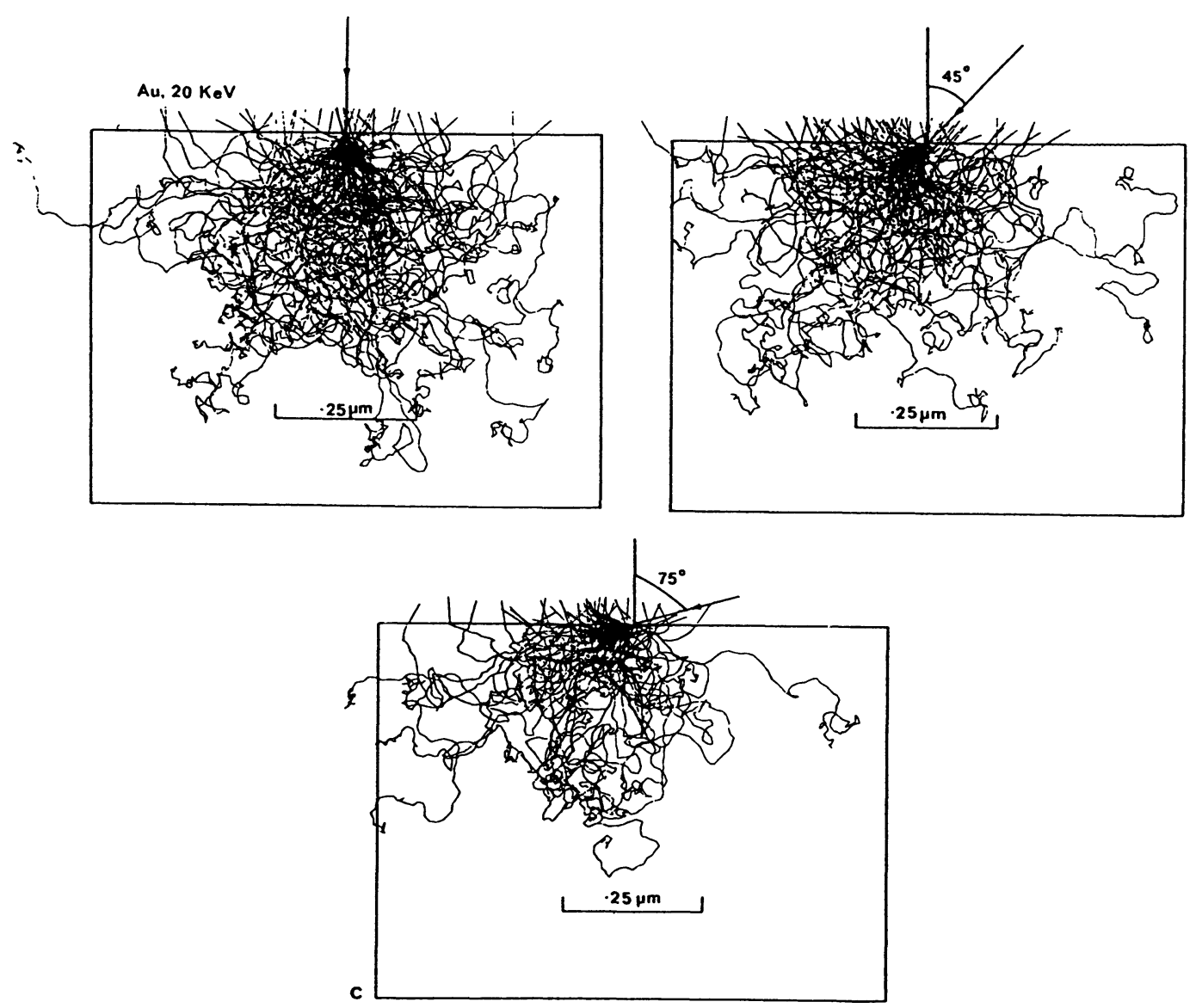

Fig. 2. - Pictorial view of the random walk of a $20 \mathrm{keV}$ electron beam into a gold sample. Angles of incidence of 0,45 and 75 degrees are shown. (Courtesy M M El Gomati).

an increase in beam current will result in an increase in beam current density for a fixed beam size and this may lead to an increase in the rate at which the sample is damaged by the electron beam. (Practical instruments use beam energies in the range $5 \mathrm{keV}$ to $100 \mathrm{keV}$, beam currents in the range $1-10 \mathrm{nA}$ and beam diameters in the range $1 \mathrm{~nm}$ to $500 \mathrm{~nm}$. Beam current densities of the order of $1000 \mathrm{~A} \mathrm{~cm}^{-2}$ are common). Therefore, one is usually faced with the need to accept long times for image acquisition.

1.3 Spatial Resolution. - Theoretical evaluation of the spatial resolution of a scanning electron probe technique can be carried out using Monte Carlo modelling of the interaction of the electrons with the sample. An example for the trajectories of $20 \mathrm{keV}$ electrons striking the surface of a copper sample at 3 angles of incidence is shown in Figure 2 . It can be seen that most of the electrons execute a random walk into the sample and finally reach their range where they contribute to the current flowing from the sample to ground. Some, however are backscattered towards the surface where they can ionise atoms to produce Auger electrons which are sufficiently near to the surface that they can escape into the vacuum and may be detected. Some electrons inside the sample will ionise atoms and characteristic X-rays will be produced. - these too can escape but from greater depths than the Auger electrons because the scattering cross-sections for 


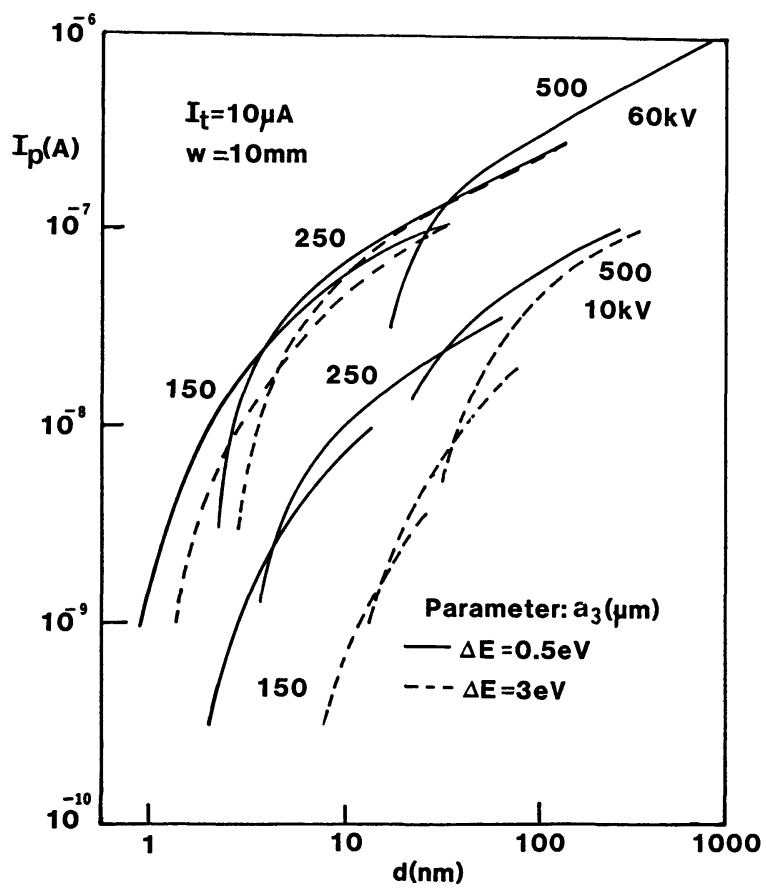

Fig. 3. - Beam current versus spot size for a particular electron column. (Courtesy Venables and Archer) The calculations are made for an emission current of $10 \mu \mathrm{A}$ with an energy spread of $0.5 \mathrm{eV}$ (solid lines) or $3 \mathrm{eV}$ (dashed lines). The calculations include a beam angle limiting aperture whose diameter is marked in mm beside each curve. Both $60 \mathrm{keV}$ and $10 \mathrm{keV}$ calculations are shown.

$\mathrm{X}$-rays are smaller than those for electrons. It can also be seen in Figure 2 that the scattering of the incident electrons is forward peaked and that the number of electrons reaching the surface rises as the angle of incidence increases. The method has been reviewed recently by Shimizu and Ding Ze-Jun [6].

The Monte-Carlo simulation of Figure 2 carried out with a stream of electrons striking the sample in an infinitesimally narrow beam. In practice the incident beam has a finite width - often regarded as having an approximately Gaussian distribution. The beam has this finite width because of the Coulomb repulsions between its electrons, because of the spherical, chromatic and astigmatism aberration effects in the components of the beam forming system and because of diffraction effects at the apertures in these electron optical components. These effects are described in detail in many textbooks on electron optics - see for example Grivet [7] and Wells et al. [8]. The net effect of these aberrations is that the current which can be focussed into a beam of electrons at a chosen energy always decreases as the apertures are modified to produce smaller beam sizes. Since a small beam size is what is required for high spatial resolution (see below) the higher the resolution required the smaller the beam current will be and the longer will be the acquisition time for data of given statistical precision. An example of the variations of beam current with beam size for a particular electron column modelled and measured by Venables and Archer [9] is shown in Figure 3.

In order to try to use as high a beam current as possible whilst maintaining a small beam diameter it is important to use the brightest source of electrons that can be found. There are two kinds of sources which have brightnesses greater than that of the simple tungsten thermionic emit- 
ter. These are lanthanum hexaboride $\left(\mathrm{LaB}_{6}\right)$ and field emission sources. $\mathrm{LaB}_{6}$ sources can have brightnesses of the order of $5 \times 10^{6} \mathrm{~A} \mathrm{sr}^{-1} \mathrm{~cm}^{-2}$ and field electron emitters based upon very sharp tips of W(100) coated with $\mathrm{Zr}$ or tips of clean W(310) can have brightnesses of the order of $10^{10} \mathrm{~A}$ $\mathrm{sr}^{-1} \mathrm{~cm}^{-2}$. The field emitters are the favoured sources for analytical electron microscopes because they have the highest brightness obtainable and they can be fabricated as Schottky [10] emitters in a form which is very reliable and can result in an electron beam which has a stable current over very long periods of time (many 100's of hours). Using such sources it is possible to generate, for example, a beam of electrons with a diameter of $50 \mathrm{~nm}$, a current of $10 \mathrm{nA}$ and an energy of $5 \mathrm{keV}$ using an all electrostatic column (Todd et al. [11]) or a beam with energy $100 \mathrm{keV}$, diameter ca 4 $\mathrm{nm}$ and current $1.5 \mathrm{nA}$ using a column with magnetic lenses, (e.g. Bleeker and Kruit [12]) which have lower spherical aberration effects than electrostatic lenses.

Monte-Carlo modelling near to an abrupt chemical edge in a flat surface can be carried out with an infinitesimally narrow electron beam striking the surface at a variety of distances away from the edge. This calculation leads to an edge resolution function for that combination of materials, angle of incidence and beam energy. The spatial resolution for a sharp edge with a practical beam of finite width can then be found by convoluting the beam intensity profile with the edge resolution function. This calculation was reported by El Gomati et al. [13] who showed that the spread of a sharp edge, $\Delta_{50}$, from $25 \%$ to $75 \%$ of the total change in Auger signal occurring across the edge is given by:

$$
\Delta_{50}=2 r b
$$

In this equation $r$ is the Auger backscattering factor and $b$ is the full width at half maximum of the Gaussian electron beam. Since $r$ is usually in the range 1 to 2.5 the edge resolution is somewhere between 2 and 5 times the beam width depending upon the atomic number of the sample. The importance of backscattering effects in the determination of the spatial resolution has been reported by Janssen and Venables [14]. The validity of equation (2) has been tested experimentally by El Gomati et al. [13] and it appears to provide a good working guide to the spatial resolution to be expected.

In order to obtain the best spatial resolution there have been two extreme approaches. One is to reduce the spreading effects of electron backscattering by reducing the primary beam energy. This was the tactic adopted by Todd et al. [11] who demonstrated a miniature electron column based upon a field emission source which focussed a beam of electrons at $5 \mathrm{keV}$ into a spot about $50 \mathrm{~nm}$ wide. At the other extreme, the primary energy can be raised to reduce the spot size by reducing the aberrations in the column. This has been demonstrated by Cazaux et al. [15] who used a STEM working at $100 \mathrm{keV}$ and by Hembree and Venables [16] using a very special electron spectrometer also in a STEM (see later). The instrument described by Hembree and Venables has demonstrated the best spatial resolution obtained to date - ca $4 \mathrm{~nm}$ at $100 \mathrm{keV}$ using small Silver particles on a Si(100) substrate - Figure 4.

The spatial resolution observed with a practical surface which may be very rough and may even contain sharp or re-entrant steps is worse than that estimated from equation (2) or observed in idealised flat surface experiments. There are a variety of complicating scattering processes occurring at such steps which can degrade the resolution. These will be discussed later.

Detailed considerations as to the physical limits applying to the spatial resolution have been presented by Cazaux [17] who predicts that chemical identification of a single atom will be possible using AES for those samples that are able to tolerate a very large electron dose.

1.4 Spectrometers in Imaging Instruments. The CMA and the CHA. - There are two kinds of electron spectrometer in widespread use in scanning Auger microscopes. They are 


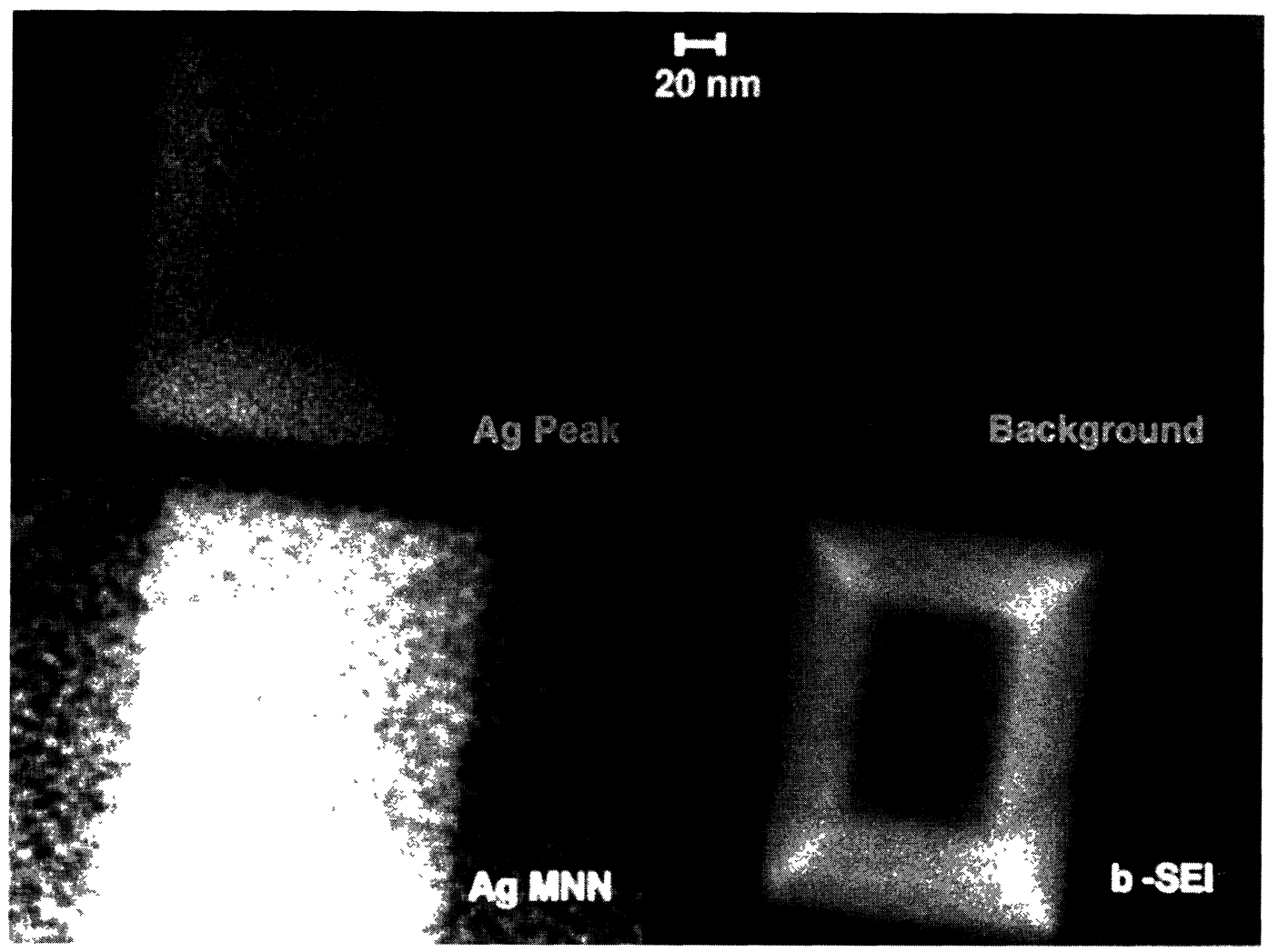

Fig. 4. - A silver crystal on a silicon (100) substrate at $500^{\circ} \mathrm{C}$ imaged in the MIDAS instrument. a) Energy analysed image on Silver peak. b) Energy analysed image on the background just above the silver peak. c) The silver MNN Auger image produced from a) and b). d) The secondary electron image of the same crystal. (Courtesy Prof Venables).

the cylindrical mirror analyser (CMA) and the concentric hemispherical analyser (CHA) which usually incorporates a transfer lens - Figure 5.

The former is attractive for its mechanical and electrical simplicity - it consists of a pair of coaxial cylinders with appropriate entrance and exit slits. Further, it can be constructed to collect a reasonably large fraction of the electrons emitted from the sample into $2 \pi$ sr. $-10 \%$ is common - which means that this spectrometer is favoured for relatively fast image acquisition. Its disadvantages are that it operates at constant energy resolving power as the spectrum is swept which means that electron counts are lost at low energies and it can have a rather small field of view the maximum area of the surface under study which can be scanned and used to form an image without unacceptable loss of transmission around the spectrometer.

The latter has the advantages that it can be operated in different modes in order to optimise the measurement of the quantity being observed. The entrance aperture may be reduced to increase the energy resolution or increased to improve the solid angle of collection of electrons leaving the sample. The potentials used may be arranged to sweep through a spectrum with constant energy resolving power or with constant energy window. With appropriate design of the transfer lens between the sample and the hemispheres the field of view can be arranged to be quite large 

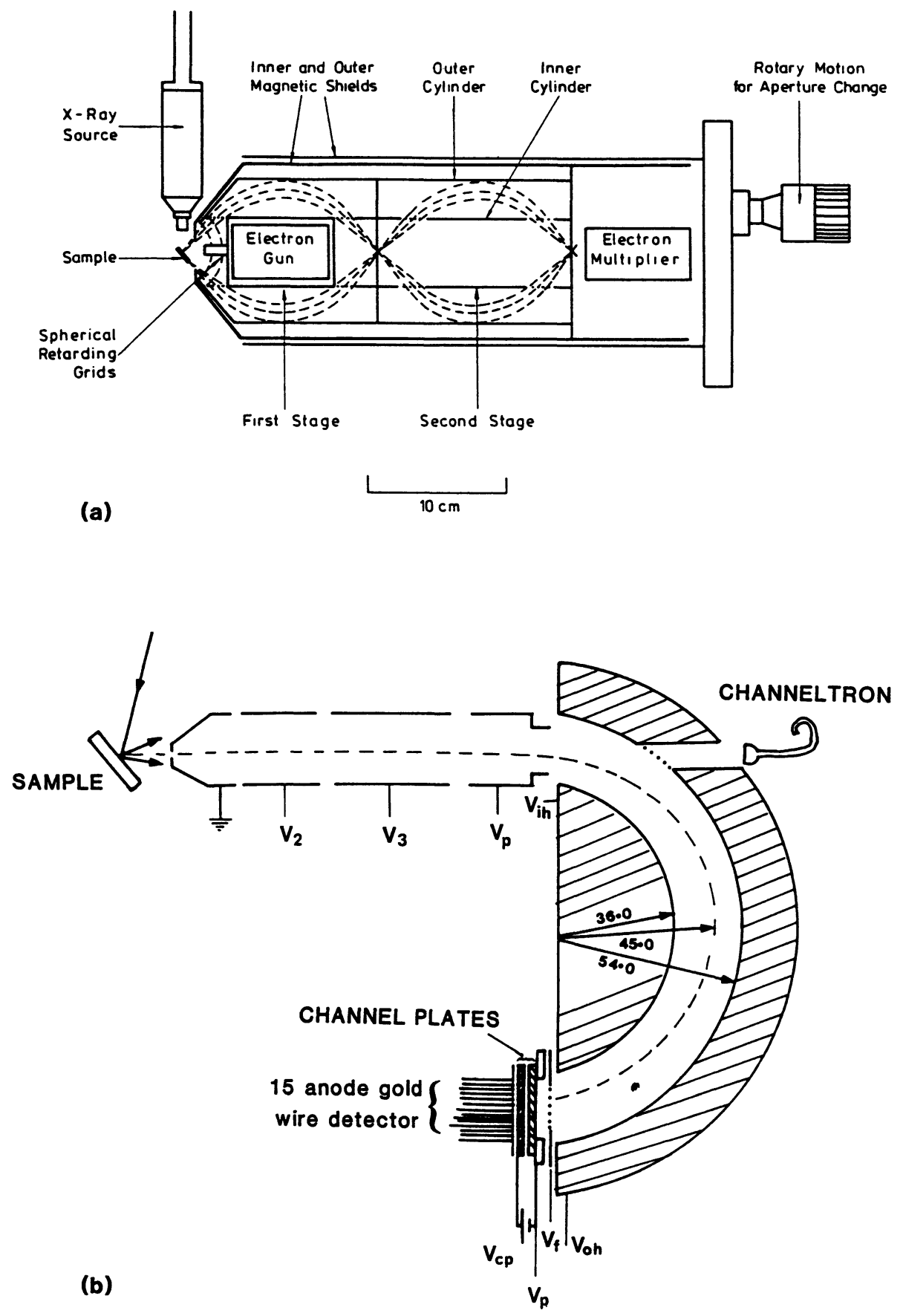

Fig. 5. - a) A double-pass cylindrical mirror analyser (CMA) which consists of two CMA's in series. (Courtesy of P W Palmberg and Elsevier Scientific Publishing Company). b) A concentric hemispherical analyser (CHA). This is the CHA in the MULSAM instrument referred to later in the text. The accelerating voltage across the channel plates is referred to the kinetic energy of the electrons leaving the sample. The radii are in $\mathrm{mm}$. 
compared to that of a CMA - perhaps a few hundred microns across. The disadvantages are that it is substantially more complex than a CMA because there are many electron optical elements whose potentials have to be varied as a spectrum is swept; the solid angle of collection is usually smaller than that of a CMA - it may be in the range $0.05-2 \%$ of $2 \pi$ sr. The flexibility of the CHA and its adaptability for multi-channel operation (see later) make it a favoured choice at the moment.

1.4.1 Transmission Functions. - The transmission function $T(E)$ of an electron spectrometer can be defined as the fraction of the number of electrons entering the entrance slit of the spectrometer with the correct energy to reach the detector which actually do reach it and are registered and counted as particles. Clearly, an ideal spectrometer would have a transmission of unity for all energies. In practice this is impossible. One reason why this is so is that the Helmholtz-Lagrange law is at work in the transfer lens between a sample and the hemispheres of a CHA. This means that as the retardation of the transfer lens is increased so as to slow the electrons to the correct energy to pass around the gap between the hemispheres so the angular divergence of electrons leaving the transfer lens must rise. This divergence can become so large that even electrons with the correct energy to be detected can strike the metal parts of the system and are lost to the detector. Thus $T(E)$ is generally a decreasing function of $E$. Secondly, the aberrations in the transfer lens also spread the beam out and allow any apertures or sections of restricted diameter to cut off some the electrons destined to reach the detector. This too results in $T(E)$ being a falling function of $E$.

Why should this falling $T(E)$ be of consequence in an imaging context? In the first place, the arguments above about signal to noise ratio have shown how the analyst is always working to keep the signal as high as possible. At higher kinetic energies the low value of $T(E)$ means that electrons are being lost in their path through the spectrometer and this represents lost sensitivity and increased frame scan times. Secondly, $T(E)$ is a falling function of $E$ because some electron trajectories intercept metal parts in the spectrometer and the extent to which this occurs is changed as the source of electrons is moved about on the surface as the primary beam is scanned. This means that $T(E)$ can change for different sample positions and is the origin of the finite field of view of a spectrometer. If the spectrometer is being used to collect images which are going to be quantitatively interpreted then this variation will add to the complexity of analysis. Finally, if the spectra obtained using the spectrometer in the microscope are to be compared with spectra obtained in other instruments then it is essential to correct the measured spectra for the distortion caused by $T(E)$ for each instrument.

A further degradation of the observed spectrum which affects the extent to which images have acceptable signal to noise ratios and can be quantitatively interpreted is due to scattered electrons inside the spectrometer structure reaching the detector and adding to the background under the peaks being measured. Electrons travelling around the centre of the gap between the hemispheres of a CHA are focussed on the detector and counted. However, electrons with kinetic energies just above this value can strike the outer hemisphere near the exit plane of the CHA (Fig. 6a). These scattered electrons will clearly contribute an amount $I(E)$ to the background under any peak in the spectrum and reduce the signal to noise ratio. This mechanism has been identified and studied by Seah and Smith [18] and extended by Greenwood et al. [19]. Secondly, electrons with energies above those focussed by the transfer lens of the spectrometer will strike metal parts on this lens and there generate secondaries (Fig. 6b). Some of these secondaries may also reach the hemispheres, pass around and be detected, also contributing to the background.

A third complication arises from the fact that the channel electron multipliers or microchannel plates, usually employed to detect individual electrons successfully navigating the spectrometer, have gains which can vary with kinetic energy of the electrons arriving at their front faces, their 


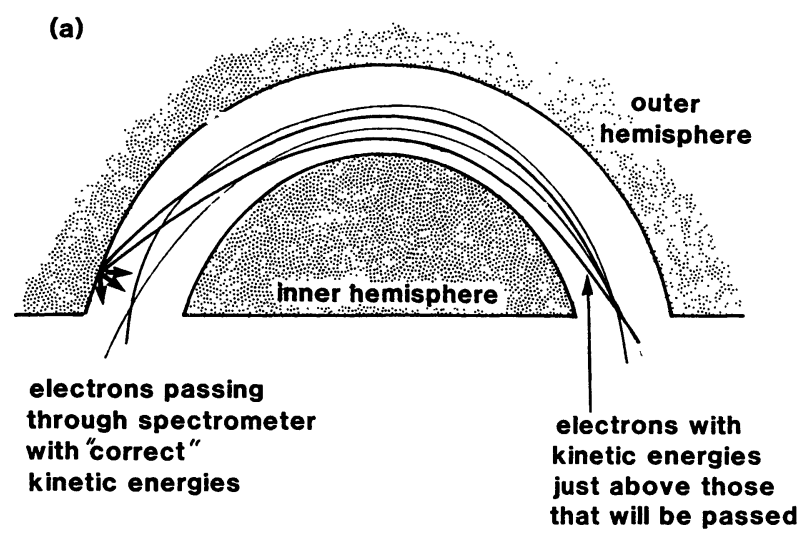

(b)

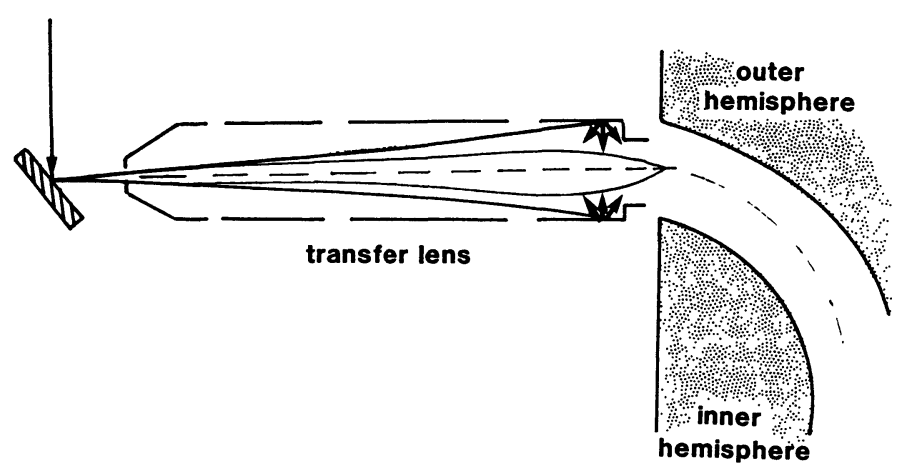

Fig. 6. - Internal scattering originating a) at the outer hemisphere and b) in the transfer lens of a CHA.

state of cleanliness and the total dose of electrons to which they have been exposed. This whole subject has been studied carefully by Seah and Tosa [20]. The kinetic energy variation can be overcome by accelerating the electrons from the exit plane of the hemispheres to the front face of the channeltron or channel plates such that they arrive with a kinetic energy independent of their initial kinetic energy. The other two effects are harder to quantify. One approach is to mount a standard elemental sample on the specimen manipulator alongside the sample under study. If this is always the same material (say gold or silver) then the area under the spectrum of the standard can be normalised to the same quantity at some defined time. This ratio can then be used to scale the spectra or pixel values of the unknown to the sensitivity at the same defined time. This is not a time consuming process and provides a useful guide as to the extent of ageing effects in the detectors.

1.4.2 Other Sensitivity Considerations - Beam Damage and Detector Dead Times. - The solid angle $\Omega$ subtended by the spectrometer entrance aperture at the sample surface determines the fraction of all emitted electrons which are collected and may be used to form a signal. Therefore $\Omega$ is a crucial parameter determining the sensitivity. As discussed above it has a maximum value which is usually determined by the energy resolution required.

Another way of raising the sensitivity is to increase the current in the incident beam (see Eq. 
1). However, this too is the subject of a balance between competing effects. First, the effect of raising the current in the beam is to increase the beam diameter because of increased Coulomb repulsions within the beam - space charge effects. Because of the arguments above this degrades the spatial resolution which can be obtained. Secondly, an increased current density in the beam can result in increased damage of the sample surface. The subject of the damage of solids by electron bombardment has been reviewed by Pantano and Madey [21]. Insulating materials like oxides and many organic materials undergo dissociation or desorption in an electron beam which, of course, causes a time dependent variation in the surface chemical composition. The damage mechanism, in many cases, appears to be dependent upon the total dose of electrons striking a point on the surface. Pantano and Madey suggest that noticeable damage to adsorbed monolayers on metals will occur with a dose of $5 \mathrm{keV}$ electrons of $1.6 \times 10^{-4}$ to $1.6 \times 10^{-3} \mathrm{C} \mathrm{cm}^{-2}$. For damage to bulk materials they suggest that noticeable damage occurs at about 10 times this dose. These figures are applicable to those materials that damage rather easily in the electron beam such as $\mathrm{Al}_{2} \mathrm{O}_{3}, \mathrm{SiO}_{2}$, alkali halides and most organic materials. Metals and semiconductors can be exposed to many orders of magnitude higher doses and not show any sign of damage.

As the rate of arrival of electrons at a channeltron or microchannel plate rises because $\Omega$ or the beam current has been increased, a new source of distortion of the spectrum can occur. This is due to the non-linearity of these detectors which exhibit a dead time effect causing the measured count rate to saturate as the incident rate is increased. This can be corrected for provided the deviation from non-linearity is not too large. The correction used in the York MULSAM instrument (see below) is of the form:

$$
n=\frac{m}{S-\tau m}
$$

In this expression $n$ is the corrected count rate, $m$ is the observed count rate, $S$ is the sensitivity of the detector and $\tau$ is the dead time for the detector. The quantities $S$ and $\tau$ are determined in a calibration experiment.

1.4.3 The Field of View of the Spectrometer. - Every spectrometer has a field of view, $S(E, x, y)$, within which electrons at energy $E$ can be detected with a sensitivity proportional to $T(E, x, y)$ for a particular range of positions $(x, y)$ of the electron beam on the surface. This is an important property when imaging because $S(E, x, y)$ determines the area of the sample that can be viewed and analysed for any particular setting of the spectrometer potentials. If the area being scanned is large (low magnifications) then the field of view can often be seen in the image contrast. The field of view of the double pass CMA has been investigated by Erickson and Powell [22] and that of a CHA system by Peacock $e t$ al. [23] with extensions to modifications of the spectrum due to electrons scattered within the spectrometer by Greenwood et al. [19]. Because of the Helmholtz-Lagrange law, the field of view of a CHA accessed by a transfer lens becomes smaller as the electron optical retardation of the transfer lens is increased. Thus, if a spectrum is scanned with constant pass energy of the electrons around the hemispheres then the field of view becomes smaller as the spectrum is swept up to higher kinetic energies. This can be a very important consideration when imaging because incorrect positioning of the sample can result in the scanned area falling outside the field of view of the spectrometer. It is important to be able to centre the field of view when using a large flat sample in the field of view before moving the sample manipulator to bring the sample under study into the same position before acquiring data which is to be quantitatively interpreted.

1.4.4 Examples of Microscope Configurations. - Three distinctive examples of UHV, energy analysing, scanning electron microscopes designed for scanning Auger work are shown in Figures 7a-c. 
(a)

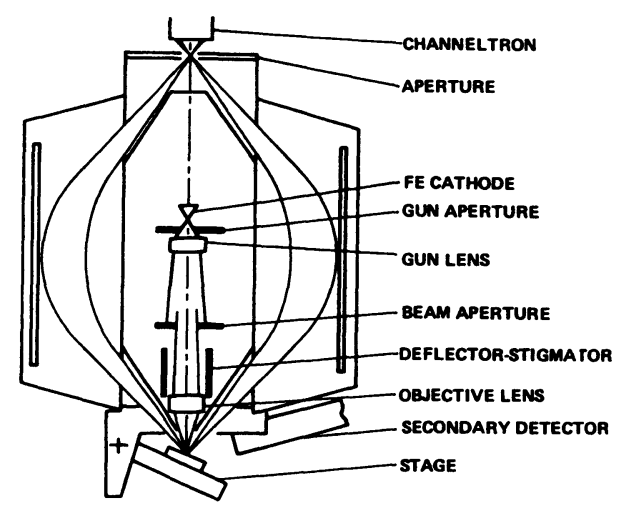

(b)

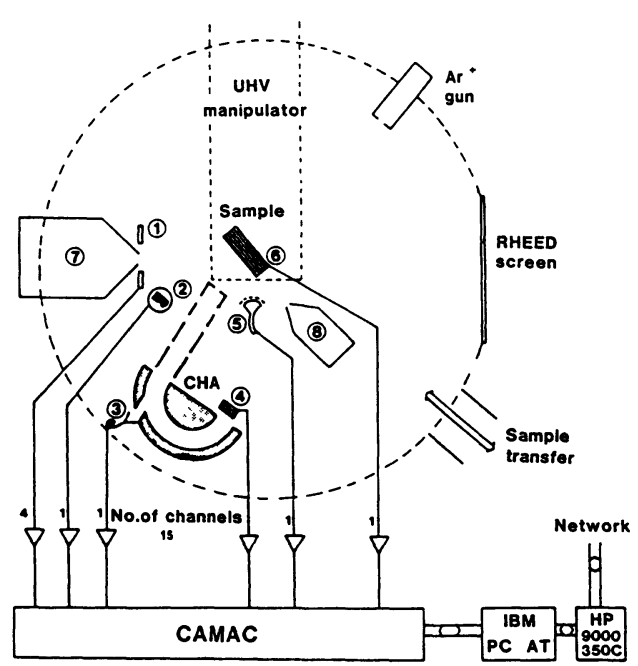

(c)

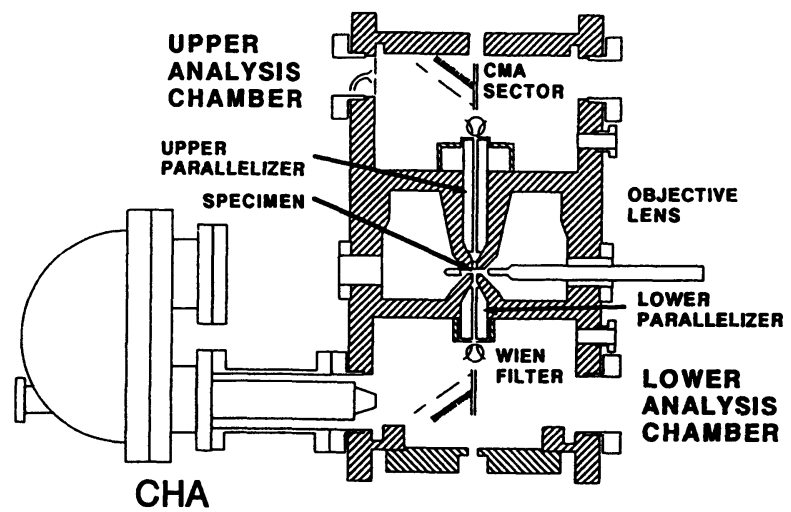

Fig. 7. - Three arrangements of components in SAM's. a) Poppa. A single pass CMA with coaxial field emission electron gun and integral specimen manipulator. (Courtesy H Poppa) b) Prutton (MULSAM). A CHA with seperate field emission gun and and array of different detectors working in synchronism. c) Venables (MIDAS). A modified with STEM with a paralleliser, CMA section as a deflector and an external CHA. (Courtesy Venables). 
The principle of Auger microscopy was first demonstrated in 1971 by Macdonald and Waldrop [24]. An early (1979) practical instrument was a compact assembly of a miniature field emission electron gun and column mounted coaxially in a CMA was described by Todd et al. [11] of Stanford University and is sketched in Figure 7a. The gun delivered a beam of about 5 nA into a spot about $50 \mathrm{~nm}$ diameter at $5 \mathrm{keV}$. The specimen manipulator was mounted directly onto the gun/CMA assembly in order to minimise the effects of mechanical vibrations. The whole assembly is supported on a single 10 inch diameter UHV flange. The instrument was subsequently placed under computer control and modified to detect and image spin polarised electrons by VanZandt et al. [25].

A different philosophy was adopted at York where emphasis was placed upon the technique required to quantify images in order to map the variations of the chemical composition in the surface. Spatial resolution was a second priority. The first instrument here was reported by Browning et al. in 1977 [26] and this developed into the instrument sketched in Figure $7 \mathrm{~b}$. The distinctive quality of this design is that there is an array of detectors around the sample which can be used to acquire sets of simultaneous images of the same region on the sample. This instrument is referred to as MULSAM or MULti-spectral Scanning Auger Microscope. The beam energy is $20 \mathrm{keV}$, the beam current is about $7 \mathrm{nA}$ and the beam focuses into a $200 \mathrm{~nm}$ diameter spot. The method of combining images from the different detectors using models of the physics of the scattering processes for each detector signal in order to derive information about the area being imaged will be described in the next chapters. This instrument has been described by Prutton et al. [27].

The microscope which has demonstrated the highest spatial resolution has been built and used by workers at the Universities of Sussex and of Arizona State and is based upon a heavily modified STEM. The instrument is sketched in Figure 7c and is known as MIDAS (Microscope for Imaging, Diffraction and Analysis of Surfaces). The arguments above have outlined how it becomes crucially important to collect as many of the scattered electrons as possible when the spatial resolution is improved. This was done by Hembree et al . [28] by using a novel electron spectrometer structure which incorporates a beam paralleliser before a CHA. The column in this instrument (MIDAS) delivers about $1.5 \mathrm{nA}$ in to a $4.3 \mathrm{~nm}$ spot at $100 \mathrm{keV}$. In order to focus the beam to this small spot size and yet to be able to collect a large fraction of the scattered electrons Venables $e t$ al. have designed a magnetic immersion lens which focuses electrons onto the sample and extracts emitted electrons to pass to the spectrometer. Part of this immersion lens structure is a magnetic field paralleliser (Kruit and Venables [29]). This device provides a field parallel to the surface normal of the sample which falls off as the reciprocal of the square of the distance from the sample. It causes emitted electrons to move in spirals about the field lines and converges them into a cone with about $6^{\circ}$ semiangle having collected almost all of those emitted.

\section{Auger Images, Data Manipulation and Image Correlations}

\subsection{Forming an Auger Image}

2.1.1 The peaks - heights, areas and shapes. - In order to construct images in which the contrast is proportional to the concentrations of the various chemical elements in the surface it would be advantageous to acquire a signal proportional to the area under each peak in the electron spectrum. In general this is rather difficult and has not been reported. In the first place, the estimation of peak area requires that a rather large set of energy analysed images is collected so that the whole of each peak is spanned by many images in the set. Secondly, there is always a secondary electron cascade underneath each peak in the spectrum and this would need to be modelled and then subtracted from the raw data. This is necessary so that the area of the peak 

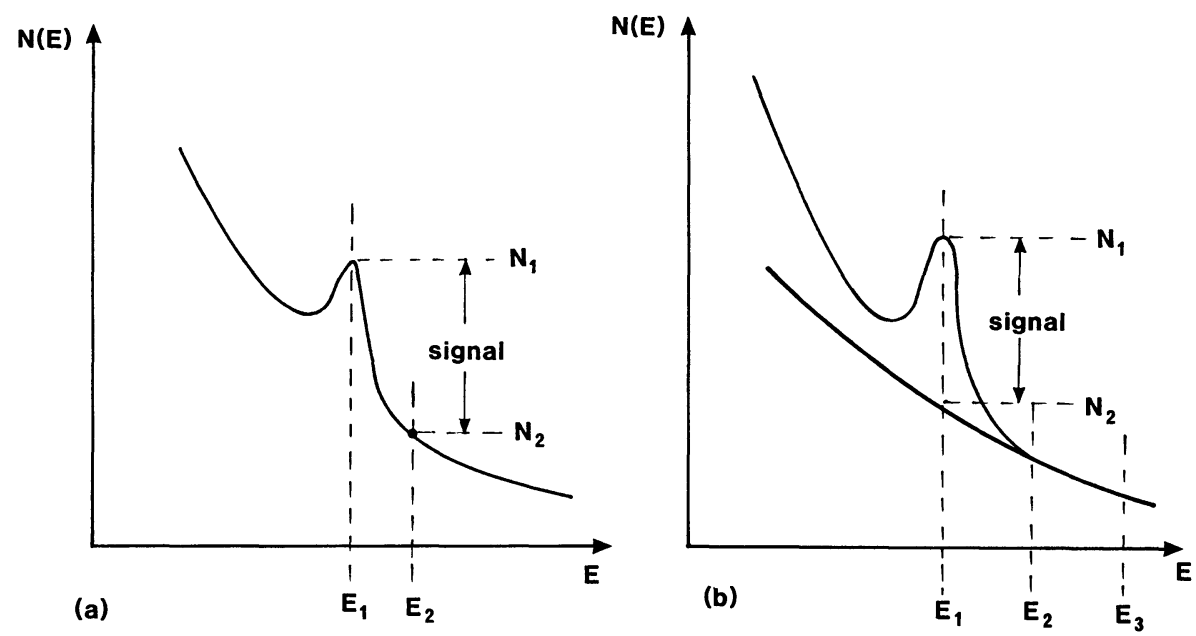

Fig. 8. - The estimation of spectral peak height. a) Peak minus background. b) Peak minus extrapolated background.

above the background can be calculated at each pixel. Therefore a great deal of data has to be acquired. Perhaps high sensitivity parallel spectrometers which collect all energies in the spectrum simultaneously will be invented in the future and this approach will become feasible. At present the acquisition times needed for this estimation of area are prohibitively large.

Instead of measuring the peak areas, several features of the peaks in the electron spectrum can be used to modulate the brightness of a digital image. The simplest to measure is the peak height which can be derived from the difference between the height of the peak, $N_{1}$, and the height, $N_{2}$, of the spectral background at some energy just above the peak (Fig. 8a). This measurement has to be made at each point on the surface which is going to be mapped into an image pixel. The procedure amounts to collecting two energy analysed images for each peak to be measured. Alternatively each peak height can be estimated by measuring the height of several points on the spectral background above each peak and then using some analytical expression to extrapolate the background $N_{2}$ at the energy of the peak. $\left(N_{1}-N_{2}\right)$ is then a signal given by the peak minus the extrapolated background height (Fig. $8 \mathrm{~b}$ ). The latter is clearly a more accurate estimate of the size of the peak but it requires the acquisition of more data - at least three measurements per pixel for linear background extrapolation. The advantages of such an extrapolation are discussed later.

The shape of the peak in the spectrum contains information about the chemical state of the emitting atoms. The simplest effect is that the Auger peaks associated with a given kind of atom are shifted in energy depending upon the chemical environment in which the atom is situated. These chemical shifts can be quite large in Auger electron spectroscopy. Thus for instance, there is a shift of $18 \mathrm{eV}$ in the energy of the $\mathrm{Mg} \mathrm{LVV}$ line between metallic $\mathrm{Mg}$ and the insulator $\mathrm{MgO}$. The Auger image may be formed with contrast to reflect this chemical shift by subtracting an image formed at the metallic $\mathrm{Mg}$ energy (45 eV) from one formed at the oxide energy $(27 \mathrm{eV})$. The detailed shape of a peak also changes (Madden [30] and Weightman in this issue) as the chemical environment changes. Another oxidation example of this kind of change is the shape of the LMM peak of metallic titanium at $416 \mathrm{eV}$ which has a sharp peaked feature at its high energy extreme. This feature vanishes rapidly as the Ti surface is exposed to small amounts of contamination particularly oxygen. Again, this change can be imaged by acquiring and post processing a pair of 


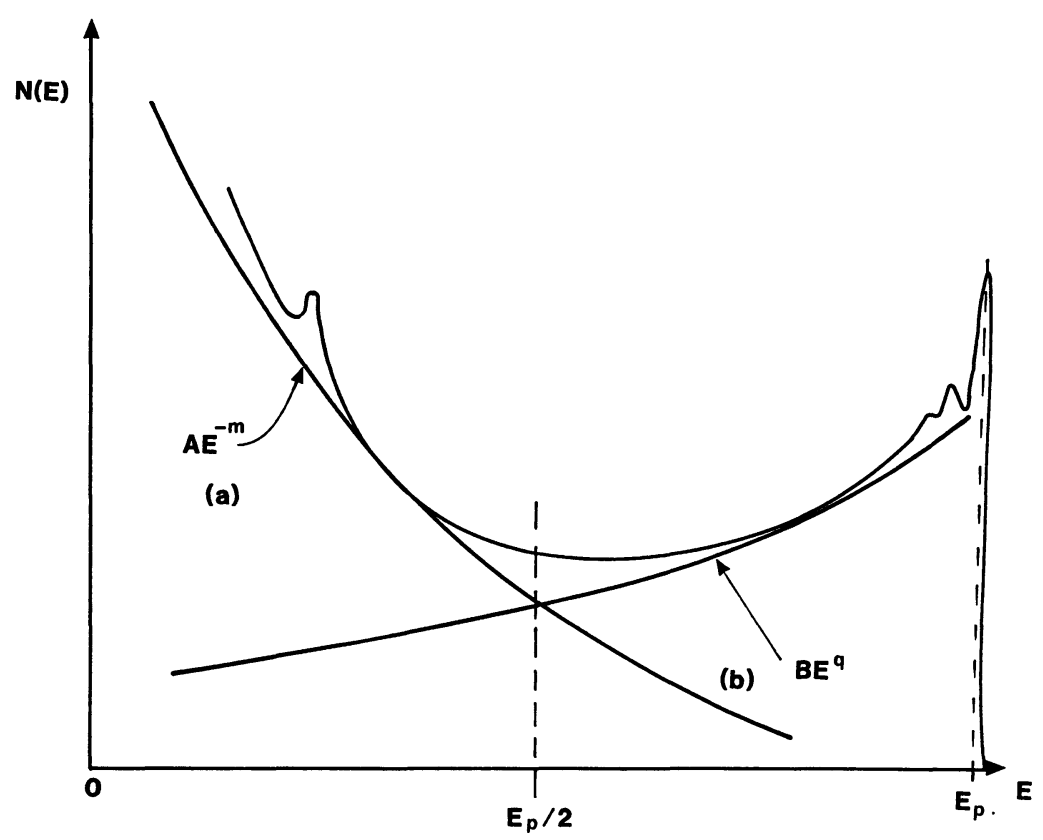

Fig. 9. - The background under a peak. a) Secondary electron cascade. b) Rediffused primary electrons.

energy analysed images.

2.1.2 The spectral background - Sickafus and $A E^{-m}$. - The shape of the spectral background in an electron excited Auger spectrum has been alluded to above. The general shape of the spectrum is sketched in Figure 9 where the curved background $B(E)$ is shown underneath the peaks. This background is important to the imaging analyst because it contributes to the statistical noise in a measurement of the peak height and because it has to be subtracted from the spectrum before a peak height or area can be estimated. In an important series of papers Sickafus [31-33] studied $B(E)$ at low primary-beam energies and found that it could be described as function of kinetic energy $E$ using the power law:

$$
B(E)=A E^{-m}
$$

In this equation $A$ and $m$ are constants of the material being studied but are functions of the primary beam energy and the angles of incidence and the take-off to the spectrometer entrance aperture. This expression is applicable for kinetic energies below about half the primary beam energy. Above this threshold the scattering of energetic electrons (often called backscattered electrons or rediffused primary electrons) can make a significant contribution to the size of $B(E)$. The contribution of backscattered electrons yields a background which is a rising function of kinetic energy. The background described by equation (1) will be referred to as the secondary electron cascade. One advantage of working with high primary energies is that the electron spectrometer is usually designed to detect electrons with kinetic energies up to a few $\mathrm{keV}$ and so the background observed is dominated by the secondary electron cascade and equation (1) is a good physical description of its shape (Matthew et al. [34]). Of course, if this is to be exploited in any background subtraction scheme it is essential to know the spectrometer functions $T(E)$ and $I(E)$ which distort 
the true spectrum and to correct the observations to remove this distortion prior to background removal operations.

If the kinetic energies to be observed are above about half the primary beam energy then another power law representation can be used and has been described by Peacock and Duraud [35]. This has the approximate form:

$$
B(E)=C E^{q}
$$

For low primary energies ( $<15 \mathrm{keV}$ ) the general form of $B(E)$ below $2 \mathrm{keV}$ is the sum of expressions (1) and (2). For high primary energies (1) is a good approximation to the background shape for the lower kinetic energies $(<2 \mathrm{keV})$ of electron emission.

2.1.3 The Signal - Removing $B(E)$ and Topography. - Approximate corrections are required for the following complicating factors:

i) the background $B(E)$ beneath each peak in the spectrum,

ii) the variation of the Auger electron yield from place to place because any real surface will be rough. This means that the angles of incidence and take-off will vary with incident beam position and the Auger yield will vary even if the concentration of the element being imaged does not vary.

iii) Given the long frame scan times discussed above there may be fluctuations from time to time in the current in the incident beam. This will result in contrast in the image unrelated to concentration variations because the Auger yield (and all other yields of emitted particles and radiation) scales linearly with incident beam current.

The simplest scheme for deriving a signal containing some correction for these effects is to calculate the peak to background ratio $P / B$ from the quantities $N_{1}$ and $N_{2}$ using $\left(N_{1}-N_{2}\right) / N_{2}$. This was proposed by Todd and Poppa [36], evaluated in a quantitative analytical context by Langeron et al. [37] and is in widespread use in commercial instruments.

Although this is a useful measure of the signal in many applications El Gomati [38] has shown that it can sometimes result in contrast artefacts due to possibility that sub-surface concentration variations can lead to variations in the signal due to changes in $N_{2}$ when there have been no changes in the surface composition.

The next simplest scheme which was devised in order to compensate for topographical effects and also corrects for beam current fluctuations is to derive the signal from two energy analysed images $N_{1}$ and $N_{2}$ but to form the ratio $\left(N_{1}-N_{2}\right) /\left(N_{1}+N_{2}\right)$. The proposal for the use of this ratio is due to Janssen et al. [39] who derived a signal from the ratio of the first derivative of $N(E)$ with respect to $E$ evaluated at the energy of the maximum positive excursion of this derivative to the value of $N(E)$ at the same energy. This is essentially a logarithmic derivative of the spectrum $N(E)$ and it is proposed as a compensator for local topographical effects because it seems reasonable to assume that the angular dependence of the background count is approximately the same as that of the peak height because they are measured at only slightly separated energies. A simple digital approximation to the logarithmic derivative is the ratio $\left(N_{1}-N_{2}\right) /\left(N_{1}+N_{2}\right)$. Prutton et al. [40] have shown that this ratio is an effective corrector for surface topography. However, $\mathrm{El}$ Gomati [38] has shown that this ratio has to be used with care because it may also overcompensate for contrast effects arising from sub-surface composition variations.

In order to correct for the variations in sub-surface composition, and the way in which they cause the slope of the background just above the peak to change from place to place, it is advantageous to 
extrapolate the background under the peak and then subtract the background at the peak energy from the peak height. 'This too was first suggested by Harland and Venables [41]. The simplest extrapolation scheme is to acquire 3 energy analysed energies at equally spaced energy intervals with one energy on the peak and at two energies on the background above the peak. In each image pixel the two background images $N_{2}$ and $N_{3}$ can be used to extrapolate linearly under the peak $N_{1}$ and to form a signal, $S$, given by:

$$
S=N_{1}-2 N_{2}+N_{3}
$$

The image contrast now has the expected sign. Of course, more accurate extrapolation schemes are possible in which, for instance, the background is measured at three energies and a quadratic function extrapolated under the peak but these schemes require more energy analysed images and so take more time to acquire. Equation (3) is not normalised in any way as are the two signals discussed previously. It could be normalised to the background under the peak by dividing the right hand side of (3) by $2 N_{2}-N_{3}$ so as to reduce the effects of beam current fluctuations.

2.1.4 Sequential Image Acquisition. - The long frame scan times associated with the serial acquisition of Auger images are common in commercial instruments which are presently available. This has tended to discourage researchers from obtaining and interpreting images in spite of the advantages outlined above. Apart from matters of inconvenience and expensive use of time, the difficulty with long acquisition times is that there may be drift both in the mechanical arrangements for holding the sample in a fixed position with respect to the electron column and in the voltages applied to the scanning electrodes which determine the position of the beam on the sample. Thus, a set of Auger images intended to be of the same region in a surface may not be spatially registered with each other. If corresponding pixels in each image of a set do not correspond to the same place on the surface then quantitative analysis of the composition at that place becomes impossible. Such images are then reduced in value because although they may indicate the spatial distribution of the chemical elements in the surface and thus be a useful guide to means for solving some particular problem they do not allow exploitation of the huge amount of information that they contained before the confusion introduced by the drift. A solution to this problem is to try to collect information from the sample in parallel rather than sequentially.

2.1.5 Parallel Image Acquisition. - A desirable solution to the problem of parallel acquisition would be to use a spectrometer which collected electrons with all energies in the spectral range required and to disperse and detect them simultaneously. No such spectrometer exists. Therefore, the best compromise that can be made at present is to collect all the electron energies required by switching the spectrometer energy rapidly whilst the beam is stationary on the surface. Thus the energy analysed images are still acquired sequentially but the time interval between the various energies at a given pixel is kept as small as possible. If drift occurs it is likely to be slow compared to the acquisition time per pixel and so the information in corresponding pixels of the images in an image set comes from very nearly the same place on the surface. Drift will cause the whole image to be distorted compared to the real surface but the spatial registration is preserved. However, other detectors - of SEM, backscattered, X-ray and other signals (Fig. 10) can be arranged around the sample and connected to electronics which does acquire the data coming from them simultaneously. Since the physics of the electron-solid interaction has been very thoroughly studied, the correlation of the energy analysed images and the images obtained from other detectors can be exploited using this physics.

Multi-spectral Auger microscopy is analogous to the LANDSAT satellite system [42] operated by NASA and the images that have been obtained from it are published in many textbooks on 


\section{EMITTED PARTICLES AND RADIATION}

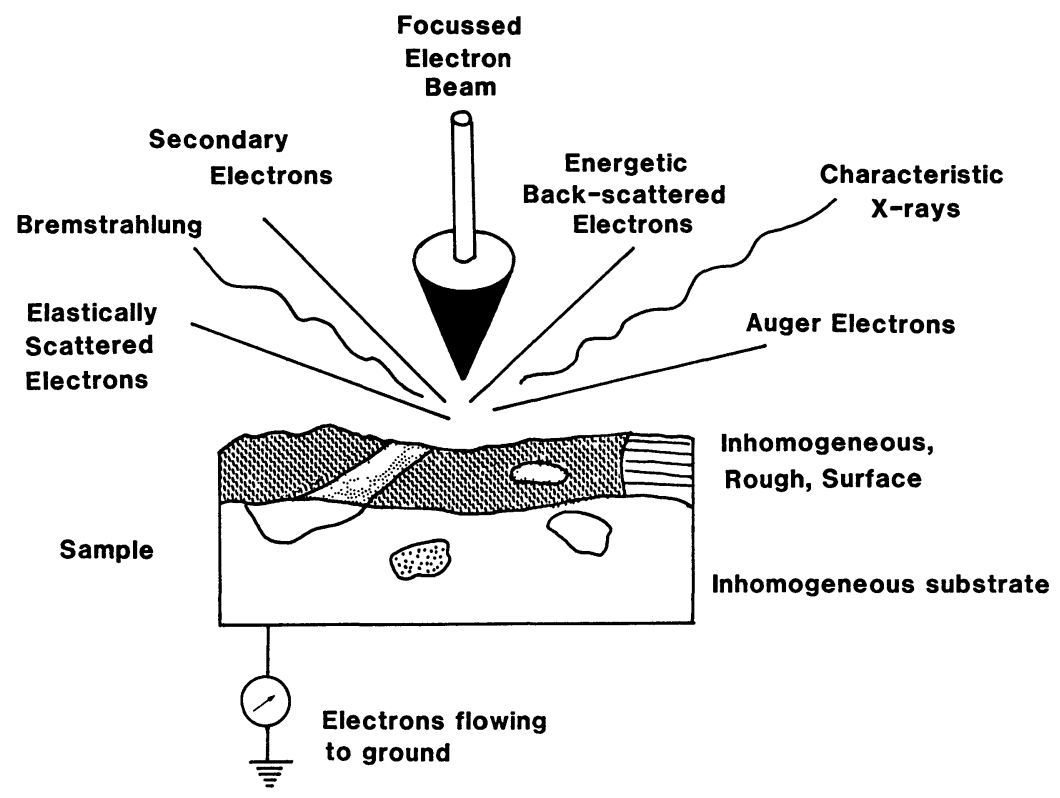

Fig. 10. - The various emissions from an inhomogeneous solid sample bombarded by energetic electrons.

digital image processing [42-44]. The idea of viewing the surface of a planet through many spatially registered telescopes each admitting a different spectral band of radiation is very similar to the idea of having several different detectors admitting different electron energies or different radiation coming from an electron bombarded surface.

The MULSAM system of analytical electron microscopy was proposed by Browning et al. [45] and has been described in detail in a series of papers by Browning $[46,47]$ and by Prutton $e t$ al. [27, 48-49]. Similar principles have also been used in SIMS by Bright and Newbury [50] (who refer to their technique as being composition histogramming), in energy dispersive X-ray imaging by King et al. [51], in STEM by Jeanguillaume [52] and in EELS by Bonnet et al. [53]. Some examples are shown below of the use of multivariate statistical techniques and of the exploitation of image correlations that become possible once spatially registered image sets are collected.

\subsection{Data Processing}

2.2.1 Scatter Diagrams. - If several spatially registered images are available from the same area on a surface then it is possible to exploit multi-dimensional histogram techniques to assist with the classification of the number of different types of region within that area. These histograms are really correlation plots but it is a convenient shorthand to refer to them as scatter diagrams. Suppose one has an image set consisting of two images obtained using two different detectors to collect simultaneous data. If the ith pixels in each image have values $N_{1}^{i}$ and $N_{2}^{i}$ then a scatter diagram is a plot in which points are marked with coordinates $\left(N_{1}^{i}, N_{2}^{i}\right)$. Thus, a pair of identical and uniform images (ie without contrast) will have all pixel pairs coincident and a scatter diagram of all the pixel pairs which will contain a single point. Two noisy, but otherwise identical and uniform images will have a scatter diagram consisting of a single cluster of points whose centre 
corresponds to the mean values of $N_{1}$ and $N_{2}$. Two perfectly correlated images will have a scatter diagram with all points on a line making an angle of $+45^{\circ}$ to the horizontal $\left(N_{1}\right)$ axis. Two perfectly anti-correlated images will show a diagram with all points on a straight line at an angle of $-45^{\circ}$. The dimensionality of the scatter diagram is equal to the number of images in the set. Finally, the number of clusters in the scatter diagram is equal to the number of different combinations of the signals there are in the image set. Thus, the number of clusters is just the number of different kinds of regions (or phases) that there are in that area of the surface. This diagram is therefore a powerful tool for making an objective decision about how many regions are needed to characterise a surface and where spectra must be acquired to estimate the composition of the region corresponding to each cluster. Examples of the use of two dimensional scatter diagrams have been given by El Gomati et al. [54] and the extension to three dimensions together with a discussion of the computational techniques involved has been given by Kenny et al. [55].

Once scatter diagrams can be constructed and displayed then false colour imaging becomes a clear possibility. Software can be written to enclose a selected cluster in the scatter diagram such that a new image is constructed which has a particular pixel colour attributed to all points which lie in that cluster. This means that the new false colour image has a contrast which identifies the different regions in the surface detectable in the original image set. Thus the new image is a phase map of the surface properties contained in the raw image set. The definition of just what is meant by a "phase" is determined by the physics of the particular signals collected by the detectors that happen to be used. This is a powerful technique often used in the presentation of images from space vehicles and some examples of its application in surface imaging are shown below.

2.2.2 Principal Component Transforms. - There are some useful statistical techniques associated with the interpretation of sets of spectra or of images which are variously known as the Hotelling Transform, the Eigenvector Transform, the Karhuenen - Loeve Transform, Factor Analysis or Principal Components Analysis (PCA). These transforms can be applied to any data that can be represented as a column vector of numbers and so they are applicable to spectra - which are clearly just tables of numbers versus kinetic energy. Images are also just tables of numbers but now they are tabulated versus the position of the beam on the sample. The methods are described in many books about image processing (loc.cit) and in books on multivariate statistics (eg Malinowski [56] and Krzanowski [57]). Gaarenstroom [58] was the first to report the use of factor analysis for electron spectroscopy and has subsequently extended it [59] to a method of quantitative analysis called target factor analysis. Applications to surface imaging have been described by Prutton et al. [48].

If all or some of the individual images in an image set are correlated with each other then there is redundant information in the set which can be exploited to improve the signal to noise ratio, increase the contrast or to reduce the total amount of information being stored. The Hotelling transform does this. The output of the transform is a set of images, the same in number as raw images but the information is compressed into a smaller set and the noise tends to be distributed in the higher members of the set. The pixel values in the transformed image set - the principal components - are linear combinations of the corresponding pixel values in the raw images and the scatter diagram is rotated about its centre of gravity. Thus, an image set containing two correlated (or anti-correlated) images would be transformed into one new principal component image containing information about the correlated components in the raw data and a second principal component image containing only noise.

This transform is useful for micro-analytical imaging because:

i) It reduces the amount of information that must be stored.

ii) The number of statistically significant principal components in the transformed set tells the analyst how many different kinds of region there are present in the solid and where they are. 
Spectroscopy of 'typical' places can be carried out subsequently to identify the differences between these regions. The selection of what is typical is then completely objective.

iii) Although the overall signal to noise ratio is unchanged as a result of applying the transform, the contrast in the first few principal component image is increased because the rotation of the scatter diagram has increased cluster separation along the principal axes.

iv) The principal component image set can reveal unexpected features of the sample surface for which specific spectral information was not sought. These regions can be analysed retrospectively to find out what is special about them.

v) The transform can be applied to data which has been pre-processed using some physical model of electron-solid scattering intended to try to separate the confusing effects of composition variations in the surface and in the bulk as well as the topography. The transformed images and their eigenvectors reveal the extent to which the pre-processing has been successful in separating the various effects at work.

Examples of some of these advantages are demonstrated below. This discussion is not confined to Auger imaging - PCA is a useful method for the examination of all kinds of spectral and image data in all the techniques outlined above. A recent paper describing the method of estimating the signal to noise ratio in an image set and applying the Hotelling Transform to EDX images has been published by Browning [60].

2.3 Image Correlations. - Some examples of the use of scatter diagrams and Hotelling Transforms are given here using data obtained using the MULSAM instrument mentioned above. There are large numbers of correlations between the signals which can be measured from the array of detectors in that system but attention will be confined here to those which help the analyst with the removal of image contrast artefacts which can prevent the accurate quantification of the surface chemical composition.

2.3.1 Backscattered Electron Signals. - The use of backscattered electron (BSE) detectors to obtain atomic number contrast ( $Z$ contrast) and topographical contrast in conventional SEM is well established [2]. A set of four quadrants of a circle of backscattered electron detectors positioned around the surface normal of a sample can be used to obtain $Z$ contrast by adding the signals from all four and topographic contrast by subtracting signals from opposing members of the four and normalising to the sum. Because the angular distribution of the backscattered electron yield is rather well understood (eg Niedrig [61, 62]) and repeatable it is possible to calibrate a set of detectors once and for all so that the local angle of incidence of the electron beam can be calculated from the positions of points or clusters of points in the scatter diagram formed from two difference images derived from the four BSE detectors [63, 64]. The local angle of incidence can be estimated from the scatter diagram of the BSE difference images using the calibration of the detectors. It should be noted that this is an experiment using correlations between four images and some physical model in order to derive the local angle of incidence map.

On the other hand, the sum of the signals from the four detectors is dominated by the $Z$ contrast in the sample. The information depth for this contrast is that corresponding to the range of the incident beam into the sample which can be from just below a micron to several microns. Therefore, the BSE sum signal can be used to derive an image in which the contrast is dominated by the variations in atomic number of the substrate with negligible contribution from a surface layer with different composition. 
2.3.2 AES/BSE Correlations. - Once the BSE detectors have been calibrated it is then possible to use them in combination with energy analysed images to produce Auger images which have been corrected for topographical artefacts'or for variations in the substrate composition. By using the difference signals from the BSE detectors an Auger correction image can be computed from a model of the angle dependence of the Auger yield. The raw Auger image is then divided (pixel by pixel) by this correction image leading to a topography corrected Auger map.

Contrast artefacts remain at sharp corners and are quite complicated to interpret and remove from the Auger maps. In both experimental and Monte-Carlo modelling studies of Auger linescans across the sharp sidewalls of etched metallic overlayers on top of silicon, El Gomati et al. [65] were able to demonstrate enhancement effects. The most extreme form of this artefact has been observed by Umbach et al. [66] who examined linescans across narrow $(0.4-1.2 \mu \mathrm{m})$ gold lines on top of silicon. The results of this study are shown in Figure 11. The enhancement of the substrate Auger signal which arises when the beam strikes the overlay near to a sidewall appears to happen because electrons are inelastically scattered in the overlay and emerge from the sidewall. They then strike the substrate both with a lower energy than that of the beam and with a more grazing angle to the surface. These two changes result in a rise in the Auger yield from the substrate above that due to the direct impingement of the beam because both the ionisation cross-section and the escape probability rise. To date there are no model calculations which simulate this effect for a general combination of materials and a general geometry. Thus correction of these edge artefacts out of Auger images is not yet possible.

The sum of the signals from the four BSE detectors can be used to derive an image in which the contrast is dominated by the fluctuations in the sub-surface atomic number. Given a model of the effect of this variation upon the Auger backscattering factor it is possible to use this $Z$ contrast image to compute a correction image to be applied to the raw Auger image. This has been demonstrated by Barkshire et al . [67] for a silicon/germanium alloy film on top of a silicon substrate in some places and top of a thick gold layer on silicon in others. The model of Ichimura and Shimizu [68] was used in this work for the $Z$ dependence of the Auger backscattering factor.

The summed BSE signals can also be used to quantify Auger depth profiles. In this case advantage is taken of the assumption that the Auger backscattering factor in a layer structure will vary in proportion to the backscattering coefficient. Since the latter is proportional to the summed BSE signal then these detectors can be used to calculate the modification to the effective Auger backscattering factor as the depth profile proceeds and this can be divided into the Auger signal at each depth to remove the broadening of the depth resolution due to substrate backscattering. This has been demonstrated by Barkshire et al. [69]. Moir et al. [70] have also reported techniques for the removal of backscattering effects from conventional depth profiles. Their method uses spectroscopic data combined with models of the scattering process rather than the image correlation procedure outlined here.

The procedures of data processing of energy analysed and backscattered electron images in order to reduce artefacts arising from topography, sub-surface composition variations and sharp edges has been reviewed recently by Prutton et al. [71] where the same sample is used to demonstrate the effects upon image contrast of the various ratios and correlations outlined above. The simple ratio methods outlined above can reduce contrast artefacts to the level of a few percent of the average image brightness but the best approach uses the same ratio but corrects it by a small factor proportional to the effective atomic number of the substrate at each pixel location [72]. This small correction can be calculated from a simultaneously acquired BSE image and seems to remove contrast artefacts to a level of the order of one percent of the average image brightness.

2.3.3 Quantitative Imaging - Pixel by Pixel Iterative Corrections. - The matrix corrections described earlier by Seah can be applied to a set of Auger images in a pixel by pixel fashion with a view to 


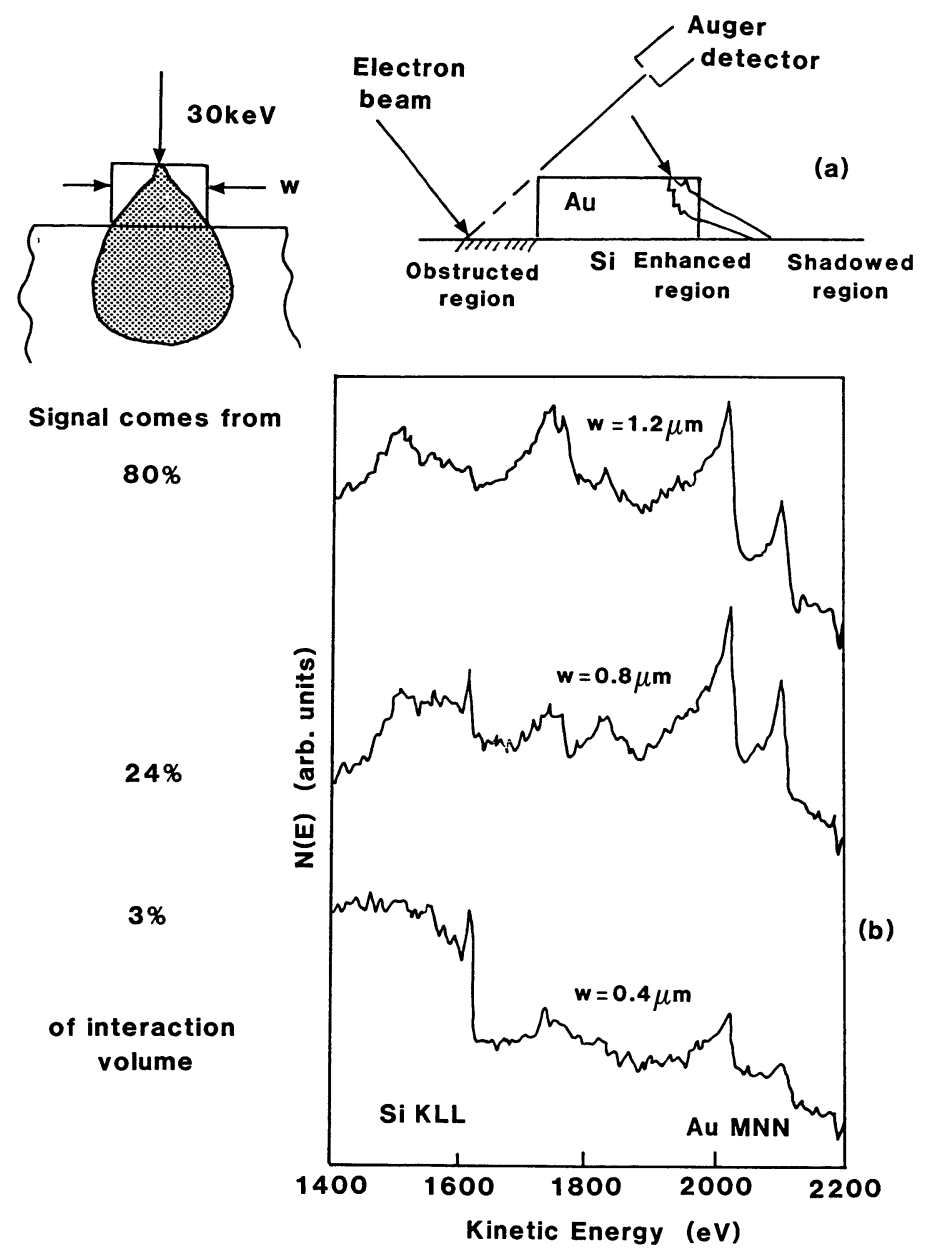

Fig. 11. - The edge effects that can occur at the sharp edges or corners of an overlay structure or very rough surface. a) Sketch depicting the origin of shadowing, obstruction and enhancement effects. b) The extreme edge enhancement observed by Umbach et al. (loc.cit) for a narrow Au strip on a Si substrate. Here, a $30 \mathrm{keV}$ electron beam str ikes a $0.6 \mu \mathrm{m}$ thick Au layer at normal incidence. Overlayer widths of $0.4-1.2 \mu \mathrm{m}$ were used as indicated. Emitted electrons entered the spectrometer with a take-off angle of $60^{\circ}$. The inset shows an overlay strip and indicates the profile of the electron beam as it enters the sample. Not only does the $\mathrm{Si}$ signal dominate the spectrum when the beam is striking only the $0.4 \mu \mathrm{m}$ Au strip but also Umbach $e t$ al. find that the spatial resolution is degraded by a factor 10. (Courtesy of Umbach et al.).

producing a new set of Auger images in which the pixel values correspond to the concentration of that element. Since the computer codes which calculate the matrix corrections usually converge very rapidly (Walker et al. [73]) this operation does not carry an excessive penalty in computing time. Of course, it does require that Auger images for each element in the surface are acquired and are spatially registered. Further, it requires that the corresponding Auger signals for bulk samples of each of the elements are available because entry into a matrix correction procedure demands that the raw Auger images be converted ints ratios of the signal from the unknown to the corresponding signal from the elemental standard for each pixel in each image. An application of 

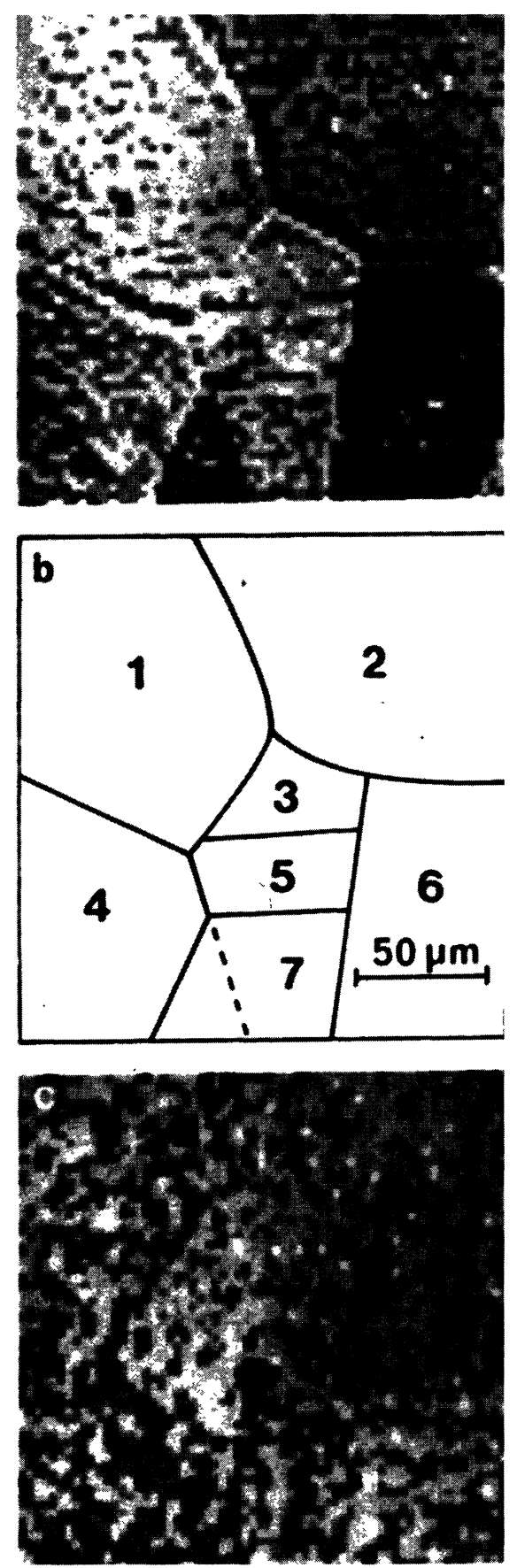

Fig. 12. - An area of a Cu-10\%Pd specimen used for a study of the anisotropy of surface segregation. a) A digital SEM image showing electron channeling contrast between grains and across a twin boundary within one grain. b) A sketch of the grains showing the labels attched to them. c) A grey scale representation of the original false colour quantitative Auger map collected after heating the specimen to $1220 \mathrm{~K}$. The grey levels represent the Pd atomic percentages varying from $7.7 \%$ (grain 4, bottom left) to ca $9 \%$ (grain 2, top right). 
this approach to quantification of surface concentration maps of $\mathrm{NiCrAl}$ alloys is given by Walker et al.

\section{Some Applications of Quantitative Surface Imaging}

This section contains a few examples of the use of surface analytical microscopies. They are chosen to illustrate the power of some of the data manipulation techniques and simultaneous image acquisition as described in the two previous chapters It is not intended to be an exhaustive survey of all the different kinds of measurements that have been made.

3.1 Surface Segregation in Alloys. - In a binary alloy surface segregation is said to have occurred when, in the absence of impurities, the surface of the material has a different composition from that of the bulk. It is of great practical importance in such fields as catalysis and corrosion where it can bring about pronounced effects upon the chemical reactions that can occur and the speeds at which they happen. Therefore it is desirable to have a theoretical model which both aids understanding and enables segregation behaviour to be predicted for previously unstudied alloys. Much of the theoretical background has been reviewed by Abraham and Brundle [74] who showed how surface segregation is driven by both the relief of bulk lattice strain and the lowering of the surface free energy. In a pair of papers Peacock $[75,76]$ described the application of Auger spectroscopy and imaging to two alloys - $\mathrm{Ni}-5 \% \mathrm{Pt}$ and $\mathrm{Cu}-10 \% \mathrm{Pd}$ - which had not been studied at the time of Abraham and Brundle's paper. They were particularly interesting binary alloys because the Abraham and Brundle theory predicted that the Ni-5\%Pt alloy should show solute (Pt) segregation but the $\mathrm{Cu}-10 \% \mathrm{Pd}$ should show solvent $(\mathrm{Cu})$ segregation. Thus, these particular alloys were in some sense a test of this theory.

Using quantification procedures similar to those described by Briggs and Seah [1], Peacock was able to show that $\mathrm{Pt}$ did indeed segregate towards the surface, the concentration rising to about 15 at $\%$ at $1050 \mathrm{~K}$. On the other hand, the $\mathrm{Cu}-10 \% \mathrm{Pd}$ alloy showed $\mathrm{Cu}$ enhancement at the surface as predicted by Abraham and Brundle. In this case the $\mathrm{Cu}$ concentration fell from its bulk value at about $930 \mathrm{~K}$ to $92 \pm 0.5 \%$ at $1300 \mathrm{~K}$. Thus, the Abraham and Brundle theory was shown to be correct for these two test materials.

Multiple simultaneous images collected from the area that had been used for the spectroscopic analysis were particularly interesting for the $\mathrm{Cu}-10 \mathrm{Pd}$ alloy. At each temperature of conducting the segregation experiment the surface composition was found to vary from grain to grain but was relatively constant across individual grain surfaces. This variation is shown in Figure 12 . It is an example of anisotropic surface segregation. In this case the chemical compositions of individual grain surfaces could be computed by averaging the pixel values over windowed image regions containing between 20 and 70 pixels. The temperature dependence of the equilibrium surface segragation could then be measured for each individual grain surface. This varied quite extremely from one grain orientation to another. One particular grain (number 6 in Fig. 12b) showed almost no temperature dependence of segregation and a 1.5 at\% enhancement of surface copper. Grain number 3, at the opposite extreme, showed a pronounced temperature dependence with surface copper changing from about $88.5 \%$ at $950 \mathrm{~K}$ to about 7.5 at $\%$ at $1300 \mathrm{~K}$.

This example is interesting in the current context because it required a multi-imaging approach and the production of quantitative Auger maps in order to obtain all the data which is required to examine the anisotropy of surface segregation from one grain to another. The high sample temperatures involved lead to considerable thermal drift in the sample position during the measurement time and simultaneous multi-imaging becomes essential in order that the pixels in the images from each chemical component of the alloy are spatially registered. The test of the theory 
would not have been feasible without a multi-imaging approach.

3.2 Superlattice Bevels and Depth Profiling. - Ion beam erosion is commonly used in combination with AES or SIMS in order to estimate the concentration distribution of elements in a solid as a function of depth. Such measurements are usually referred to as depth profiles. One way of using a scanned ion beam to obtain a depth profile is to cut a shallow angle bevel into the solid (Skinner [77]). The bevel can be imaged subsequently using SEM and MULSAM techniques, features of interest can be selected and spectroscopy or linescans carried out using measurement times appropriate to the data statistics required for analysis. This is a particularly powerful technique for analysis of materials containing thin layers such as are found in many quantum well and superlattice structures - it is one of the methods of beginning in the developing field of three dimensional characterisation of surface structures. If the angle between the bevel and the free surface is kept very small $(0.1-1 \mathrm{mrad}$ is possible in practice) then the depth information is spread out laterally on the bevel surface and so extremely high depth resolution can be obtained with moderately large electron beam diameters. The technique has been demonstrated for such layer structures by Tatlock et al. [78] and by Prutton et al. [79].

An example of an Auger image set from a superlattice containing InP and InGaAs layers which are respectively $35.6 \mathrm{~nm}$ and $9.6 \mathrm{~nm}$ thick is shown in Figure 13. In this case the bevel angle was $0.2 \mathrm{mrad}$ and the depth resolution obtained was about $2 \mathrm{~nm}$. The intrinsic resolution determined by the beam size and the bevel angle was $0.4 \mathrm{~nm}$ and so the observed resolution probably originates from ion beam mixing effects occurring when the bevel was being cut with $2 \mathrm{keV} \mathrm{Xe}^{+}$ions. The scatter diagrams in Figure 13d and e show the anticorrelation expected (from the known bulk compositions) between the phosphorus and arsenic Auger signals as well as the correlation between the indium and phosphorus signals.

This example is chosen not only because the depth resolution is good but also because this a case of the kind mentioned above in which the contrast between the layers is barely perceptible in SEM at $20 \mathrm{keV}$. Thus, the layer structure could easily have been missed if the prelimenary study of the sample was only by SEM. The Auger images could be acquired in about $10 \mathrm{~min}$ and show clear contrast corresponding to the layer structure.

3.3 Metal-Semiconductor Contacts. - The transition metal silicides, in particular those of titanium and cobalt, have been of some technological interest for their use as conductors in the interconnect patterns of VLSI circuits. These compounds are formed as thin layers on the surface a silicon device structure by rapid thermal annealing (RTA). The objective is to grow thin layers of the disilicides $\mathrm{CoSi}_{2}$ and $\mathrm{TiSi}_{2}$ which are known to have high (metallic) electrical conductivities.

An example of an ion bevelled section cut through a cobalt/silicon layer structure is shown in Figure 14. This data is described by Greenwood et al. [80]. Analysis of this bevel reveals that between the cobalt layer on top of this structure and the silicon substrate there are two other layers. Immediately beneath the surface $\mathrm{Co}$ there is a layer of uniform composition $\mathrm{Co}_{2} \mathrm{Si}$. Between this layer and the silicon there is a layer of varying composition. $\mathrm{CoSi}_{2}$ is not apparent anywhere in the structure.

An example of false colour imaging is shown in Figure 14f using this sample. The layer of varying composition contained a plasmon loss peak below the Co LMM peak which was at the silicon loss energy but enhanced in height compared to pure silicon. This peak height has been used together with the Si KLL Auger peak height to produce the false colour image. The thickness of this region of enhanced plasmon intensity is less than the layer thicknesses and is localised near to the silicon surface. Perhaps there is some reduction in the localisation of the electron dynamics of the $\mathrm{Si}$ near to the $\mathrm{Si} / \mathrm{CoSi}$ interface when there are Co atoms in the environment. This needs 


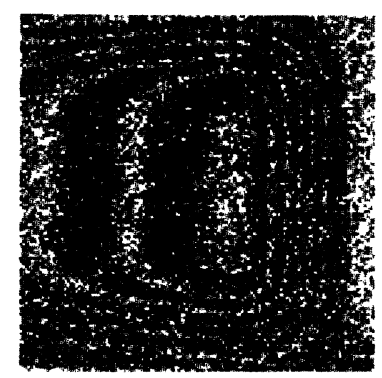

(d) As $1220 ; 1265 \mathrm{eV}$

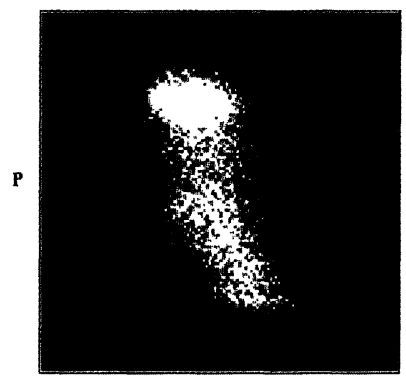

(d)
As

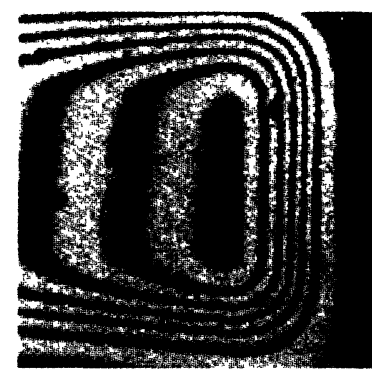

b) P 110;140 eV

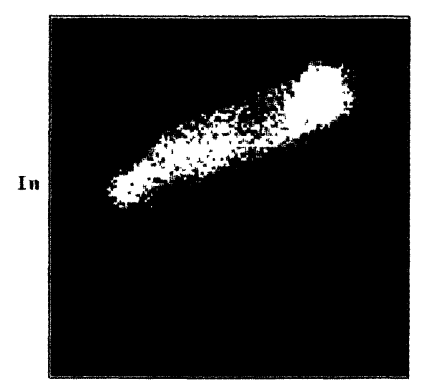

(e)

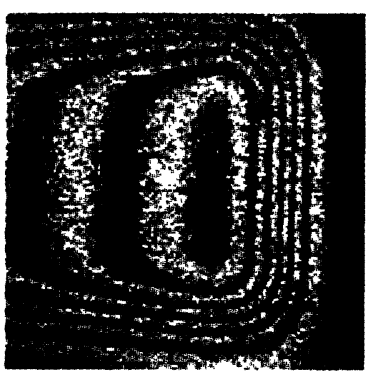

(c) In 397;430 eV

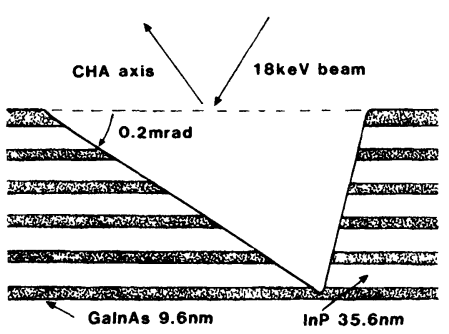

HI

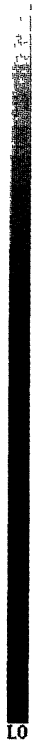

Fig. 13. - $N_{1}-N_{2}$ images for As, $\mathrm{P}$ and In in an MBE-grown structure (kindly provided by Skinner, GECMarconi Materials Development Ltd, Caswell). Beam energy $18 \mathrm{keV}$; Beam current $6 \mathrm{nA}$; constant pass energy $100 \mathrm{eV}$; Dwell times are $200 \mathrm{~ms}$ per pixel for a) and $100 \mathrm{~ms}$ per pixel for b) and c). The magnification marker is $150 \mu \mathrm{m}$ long. Two scatter diagrams and a sketch of the cross-section of the layers are shown below the Auger images.

further investigation.

The whole subject of metal (or metal-like)/semiconductor interfaces is particularly appropriate for SAM methods because the analyst is examining the majority components $(>1$ at $\%)$ of the materials so that the sensitivity of AES is adequate, spatial resolution is appropriate to the scale of device structures, quantification is practical and yet the electron beam damage effects are not too extreme.

A number of examples of the MULSAM method applied to metal/semiconductor systems are given in Prutton et al. [49] and $\mathrm{El} \mathrm{Gomati} \mathrm{et} \mathrm{al.} \mathrm{[81]} \mathrm{and} \mathrm{more} \mathrm{conventional} \mathrm{Auger} \mathrm{microscopy}$ has been used in this area and some examples are described by Oechsner [82]. In characterising semiconducting device structures the problems associated with contrast artefacts associated with quite extreme topographies become very important. The sidewalls of multilayer metallic overlays forming the circuits joining devices in VLSI are particularly important because, for instance, they may be contaminated during a fabrication process or they may be the pathways for diffusion or electromigration and so may result in eventual device failure. If contrast artefacts are present in the Auger images of the various elements then it is difficult for the analyst to decide whether or not the overlays have the structure intended. One approach to reduce this difficulty is the 

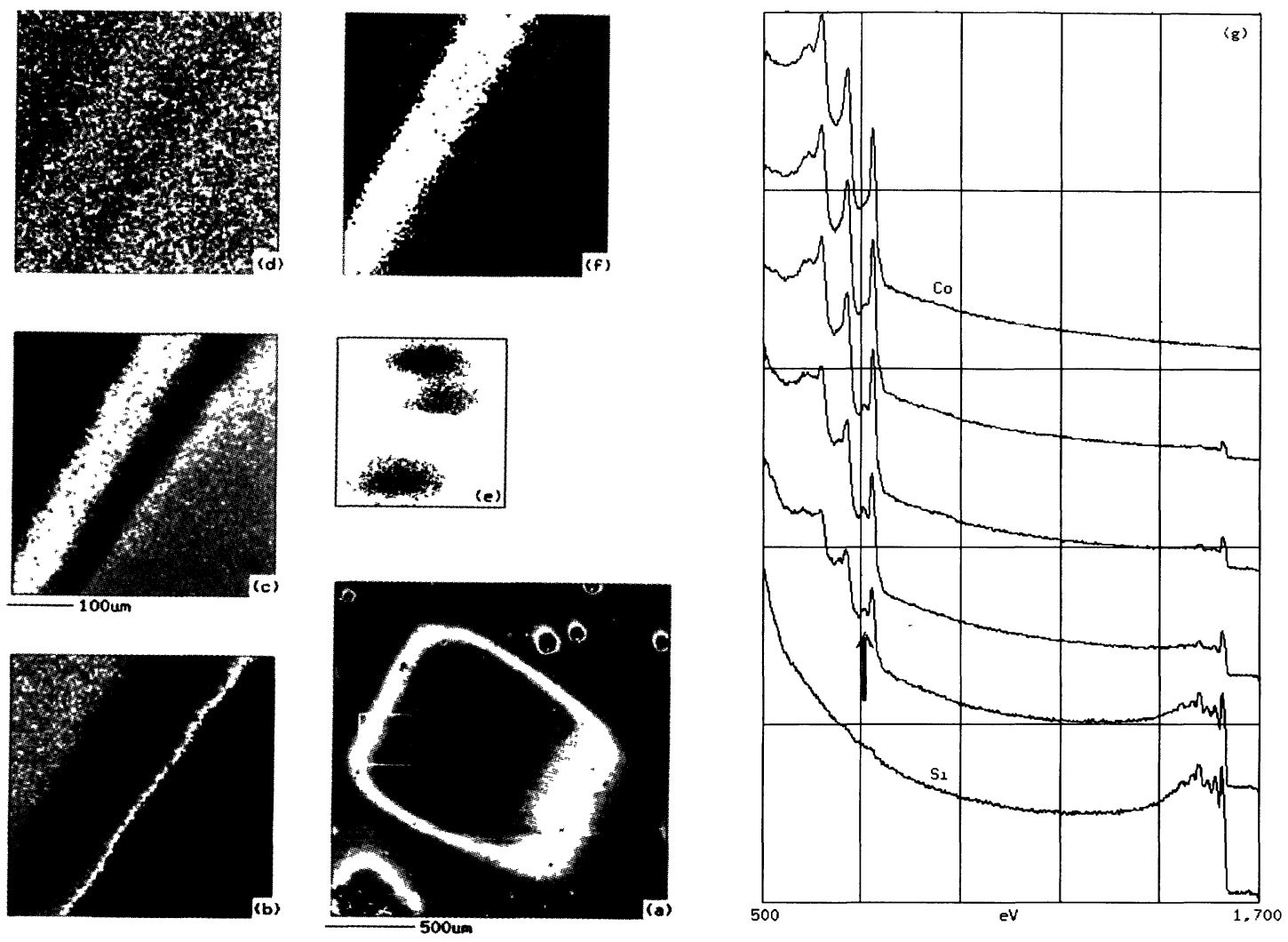

Fig. 14. - Ion beam bevel section of a Co/Si structure after rapid thermal annealing. a) An SEM image of the whole bevel. The energy analysed images were obtained from the area marked with a white square. b) Co MVV Auger image. c) Si KLL Auger image. d) A image of the same area obtained using the loss peak marked with an arrow in $\mathrm{g}$ ). e) The scatter diagram of the loss image (horizontal) versus the Si image (vertical). f) A grey scale representation of a false colour image obtained using the four cluster in e). $\mathrm{g}$ ) Spectra from different regions along a line perpendicular to the lines of contrast in $f$ ).

MULSAM philosophy of seeking to exploit image correlations in order to minimise the contrast due to artefacts. Another approach, described by Hosler [83], is to modify the acceptance angle of a CMA spectrometer with an ingenious slit arrangement. The sample is inclined to the incident electron beam so that the sidewalls of overlay structures are illuminated. A knife- edge shield is installed between the sample and the spectrometer so as to allow analysis only of those electrons emitted from the sidewall in directions nearly parallel to the plane of cicuit slice. With an angular aperture width of about $10^{\circ}$ adequate sensitivity could be retained for spectroscopy and imaging of the sidewalls and yet the contrast ertefacts could be reduced by about a factor 5 .

3.4 WEAR AND LUBRICATION. - When metallic samples are subjected to wear testing, with or without the presence of a lubricant, the surface is badly roughened with long and deep scratches in the direction of the rubbing action. One interesting problem for a tribologist is to measure the distribution of surface contaminants from the metal surfaces and the lubricant both before 


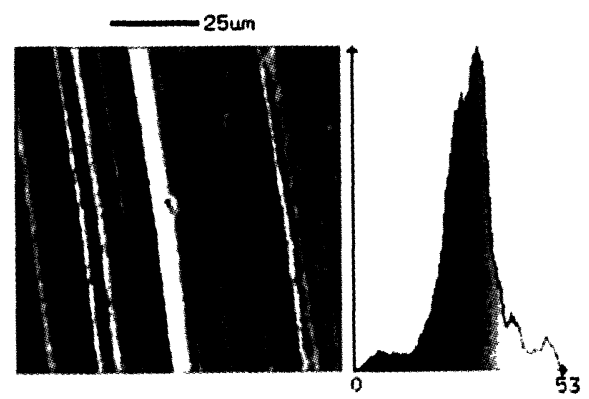

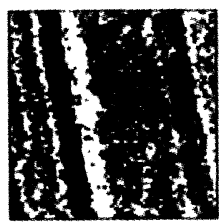

a) C Auger

ANGLE OF INCIDENCE
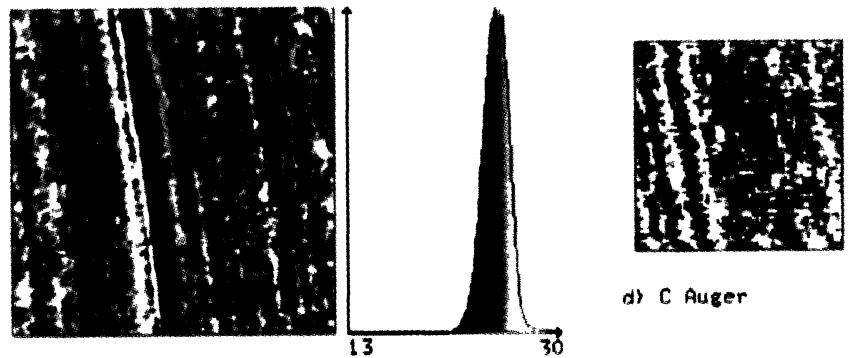

d) $C$ Auger

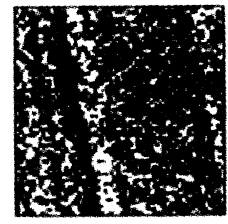

b) Ca Auger

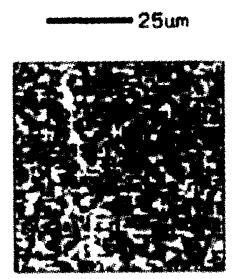

a) Co Auger

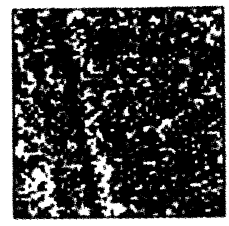

c) In Auger

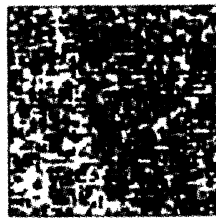

f) Zn Auger

EFFECTIVE ATOMIC NUMBER

Fig. 15. - Images from the York MULSAM instrument of the worn surface of Fe sample. The angle of incidence, $\theta$, and effective atomic number, $Z$, images on the left were obtained from the normalised differences and the sums of the four images from 4 quadrants of a backscattered electron detector placed around the $20 \mathrm{keV}$ electron beam. The contrast in these two images is quantitatively related to $\theta$ and $Z$ as can be seen from the horizontal axes of the histograms of the intensity distributions beside each figure. The Auger images a)-c) formed from the $\mathrm{C}, \mathrm{Ca}$ and $\mathrm{Zn}$ peaks respectively are uncorrected for topography and those in d)-f) are corrected.

and after the wear process. This is clearly a problem that ought to be tackled using surface spectroscopy and imaging but which is made particularly difficult by the well developed roughness in such samples.

An example of the use of BSE detectors to measure the directions of the local surface normals and the effective atomic numbers of the sub-surface material for an Fe sample after a wear test has been reported by Barkshire et al. [84] and is shown in Figure 15. The intensity histograms associated with these images show how the angle of incidence varies over the range 0 to $53^{\circ}$ this region of the sample but that the effective atomic number varies over quite a narrow range. Auger images on $\mathrm{C}, \mathrm{Ca}$ and $\mathrm{Zn}$ before (15a-c) and after (15d-f) correction with the BSE/Auger image correlation technique outlined above are shown in the same figure. It is clear that the uncorrected images show the same geometry as the scratches seen so markedly in the angle of incidence image. Careful examination of the corrected Auger images and their associated scatter diagrams (not shown here) reveals that, although the topographical contrast has not been completely removed for this extremely rough sample, there is correlation between local $\mathrm{Ca}$ and $\mathrm{Zn}$ distributions and anti-correlation between the $\mathrm{Ca}$ and $\mathrm{C}$ and the $\mathrm{Zn}$ and $\mathrm{C}$. This information was not retrievable from the uncorrected Auger images for which the amounts of all elements appear as if they are correlated with each other because the topographical contributions to the intensity dominate the contrast mechanism. 
3.5 ARCHAEOLOGY. - An unusual and interesting application of Auger imaging and spectroscopy has been reported by Paparazzo et al. [85] who examined the pipes used in ancient Rome for the distribution of water. These lead pipes or 'fistulae' were joined by an early version of soldering described by Plinium [86]. The solder, called 'tertiarium', contained about $30 \%$ by weight of Sn and modern surface analytical techniques were used by Paparazzo et al. to examine the effects of the tertiarium on corrosion at the joint. Auger microscopy proved to be useful in the identification of various oxides and chlorides in the joint as well as distinct patchiness of $\mathrm{Cu}$ and $\mathrm{Sn}$ in a rather homogeneous $\mathrm{Pb}$ background. This work is beginning to resolve a number of archaeological issues about the methods used for ancient Roman technology.

\section{Acknowledgements}

The author has been very fortunate to work with many talented people who have contibuted to the material described here in all sorts of important ways. Special thanks go to Ray Browning and Dave Peacock who, together with the author, started down the multi-imaging path. To Mohammed $\mathrm{El} \mathrm{Gomati}$ for his long standing positive collaboration and his help and ideas with many aspects of what is reported here. To Chris Walker and Peter Kenny for their ingenuity, persistence and skills with the specification, invention, and hard graft of creating the thousands of lines of computer code needed to do this kind of work. To John Greenwood and Ian Barkshire for their efforts in making many of the detectors in MULSAM, devising ways to make them really work well and for obtaining many of the fascinating first results concerning correlations between images. To Ron Roberts who, in several long visits to York from Australia, contributed to the careful and thorough characterisation of the whole MULSAM instrument and imbued everyone with a c lear idea of how to be a first class experimentalist (even if they cannot quite get there). To Jack Dee who has provided so much technical support in the design of the instrument and keeping it all running. To the technical staff of the Physics Department at York who have suffered my persistent pressures and worrying and responded with beautifully made electronic and mechanical components. The work at York has been supported with the financial assistance of first the Alvey Project and then the Silicon Towards 2000 initiative of the SERC and the DTI whose help is gratefully acknowledged. This article has used material in a longer article by the author produced as part of the proceedings of a Scottish Universities Summer School 'Quantitative Microbeam Analysis' edited by A.G. Fitzgerald, B.E. Storey and D. Fabian and published in 1993 by the Institute of Physics, Redland Way, Bristol. The author is grateful to the Institute of Physics for their permission to use their copyright material.

\section{References}

[1] Briggs D. and Seah M.P., Practical Surface Analysis Volume 1 - Auger and X-Ray Photoelectron Spectroscopy. (Wiley, Chichester, 1990).

[2] Reimer L., Scanning Electron Microscopy. Physics of Image Formation and Microanalysis. (SpringerVerlag, Berlin, 1985).

[3] Turner D.W. and Plummer I.R., J. Phys. E: Sci. Instrum. 22 (1989) 593.

[4] Krivanek O.L., Delby N., Gubbens A.J., Kundmann N.K., Leber M.I., Ray D.A. and Truong K.V., Proc. XIIth International Congress for Electron Microscopy 2 (San Fransisco Press, 1990) 76.

[5] Bishop H.E. and Riviere J.C., J. Appl. Phys. 40 (1969) 1740.

[6] Shimizu R. and Ding Ze-Jun, Rep. Progr. Phys. 55 (1992) 487. 
[7] Grivet P., Electron Opt. (Pergamon Press, Oxford, 1972).

[8] Wells O.C., Boyde A., Lifshin E. and Rezanowich A., Scanning Electron Microscopy (McGraw-Hill, New York, 1974).

[9] Venables J.A. and Archer G.D., Electron Microsc. 80 (The Hague) 1 (1980) 54.

[10] Danielson L.R. and Swanson L.W., Surf. Sci. 88 (1979) 14.

[11] Todd G., Poppa H. and Veneklasen L., Thin Solid Films 57 (1979) 213.

[12] Bleeker A.J. and Kruit P., Proc. XIIth International Conf. for Electron Microscopy 2 (1990) 380 (San Fransisco Press).

[13] El Gomati M.M., Janssen A.P., Prutton M. and Venables J.A., Surf. Sci. 85 (1979) 309.

[14] Janssen A.P. and Venables J.A., Surf. Sci. 77 (1978) 351.

[15] Cazaux J., Surf. Interface Anal. 14 (1989) 354.

[16] Venables J.A. and Hembree G.G., Inst. Phys. Conf. Ser. No. 119: Sect. 1 (1991) 33.

[17] Cazaux J., Surf. Sci. 140 (1984) 85.

[18] Seah M.P. and Smith G.C., Surf. Interface Anal. 17 (1991) 855.

[19] Greenwood J.C. Prutton M. and Roberts R.H., Surf. Interface Anal. 20 (1993) 891.

[20] Seah M.P. and Tosa M., Surf. Interface Anal. 18 (1992) 240.

[21] Pantano C.G. and Madey T.E., Appl. Surf. Sci. 7 (1981) 115.

[22] Erickson N.E. and Powell C.J., Surf. Interface Anal. 9 (1986) 111.

[23] Peacock D.C. Prutton M. and Roberts R.H., Vacuum TAIP 34 (1984) 497.

[24] MacDonald N.C. and Waldrop J.R., Appl. Phys. Lett. 19 (1971) 315.

[25] VanZandt T., Browning R., Helms C.R., Poppa H. and Landolt M., Rev. Sci. Inst. 34 (1989) 30.

[26] Browning R., Bassett P.J., El Gomati M.M. and Prutton M., Proc. Roy. Soc. London A357 (1977) 213.

[27] Prutton M., Walker C.G.H., Greenwood J.C., Kenny P.G., Dee J.C., Barkshire I.R., Roberts R.H. and El Gomati M.M., Surf. Interface Anal. 17 (1991) 71.

[28] Hembree G., Luo F.C.H. and Venables J.A., Proc. XIIth International Conference for Electron Microscopy 2 (1990) 382 (San Fransisco Press)

[29] Kruit P. and Venables J.A., Ultramicroscopy 25 (1988) 183.

[30] Madden H.H., Surf. Sci. 126 (1983) 80.

[31] Sickafus E.N., Phys. Rev. B 16 (1977) 1436.

[32] Sickafus E.N., Phys. Rev. B 16 (1977), 1448.

[33] Sickafus E.N. and Kukla B., Phys. Rev. B 19 (1977) 4056.

[34] Matthew J.A.D., Prutton M., El Gomati M.M. and Peacock D.C., Surf. Interface Anal. 11 (1988) 173.

[35] Peacock D.C. and Duraud J.P., Surf. Interface Anal. 8 (1986) 1.

[36] Todd G. and Poppa H., J. Vac. Sci. Tech. 15 (1978) 672.

[37] Langeron J.P., Minel L., Vignes J.L., Bouquet S., Pellerin F., Lorang G., Ailloud P. and Le Hericy J., Surf. Sci. 138 (1984) 610.

[38] El Gomati M.M., Matthew J.A.D. and Prutton M., Appl. Surf. Sci. 24 (1985) 147.

[39] Janssen A.P., Harland C.J. and Venables J.A., Surf. Sci. 62 (1977) 277.

[40] Prutton M., Larson L.A. and Poppa H., J. Appl. Phys. 54 (1983) 374.

[41] Harland C.J. and Venables J.A., Ultramicroscopy 17 (1985) 9.

[42] Moik J.G., 1980 Digital Processing of Remotely Sensed Images (NASA Special Publications, Washington DC U.S.A).

[43] Gonzales R.C. and Wintz P., 1977 Digital Image Processing (Addison-Wesley London).

[44] Pratt W.K., 1978 Digital Image Processing (Wiley-Interscience New York).

[45] Browning R., Peacock D.C., Prutton M. and Walker C.G.H., 1984, Inst. Phys. Conf. Ser. No. 68, EMAG83 127 (Institute of Physics London).

[46] Browning R., J. Vac. Sci. Tech. A2 (1984) 453. 
[47] Browning R., J. Vac. Sci. Tech. A3 (1985) 1959.

[48] Prutton M., El Gomati M.M. and Kenny P.G., J. Electron Spectros. Rel. Phen. 52 (1990) 197.

[49] Prutton M., Barkshire I.R., El Gomati M.M., Greenwood J.C., Kenny P. G. and Roberts R.H., Surf. Interface Anal. 18 (1992) 295.

[50] Bright D.S. and Newbury D.E., Anal. Chem. 63 (1991) 243A.

[51] King P.L., Browning R., Paque J.M. and Pianetta P., Proc. XIIth Int. Congress Electron Microsc. 1 (1990) 464.

[52] Jeanguillaume C., J. Microsc. Spectrosc. Electron. 10 (1985) 409.

[53] Bonnet N., Colliex C., Mory C. and Tence M., Scanning Electron Microsc. Suppl. 2 (1988) 351 (Scanning Microscopy International Chicago AMF O'Hare, U.S.A.)

[54] El Gomati M.M., Peacock D.C., Prutton M. and Walker C.G.H., J. Microsc. 147 (1986) 149.

[55] Kenny P.G., Barkshire I.R. and Prutton M., Ultramicroscopy 56 (1994) 289.

[56] Malinowski R., 1991 Factor Analysis in Chemistry (Wiley).

[57] Krzanowski W.J., 1988 Principles of Multivariate Analysis (Oxford University Press).

[58] Gaarenstroom S.W., Appl. Surf. Sci. 7 (1981) 7.

[59] Gaarenstroom S.W., Appl. Surf. Sci. 26 (1986) 561.

[60] Browning R., Surf. Interface Anal. 20 (1992) 495.

[61] Niedrig H., J. Appl. Phys. 53 (1982) R15.

[62] Niedrig H., 1983 Electron Beam Interactions with Solids 51 SEM Inc AMF O’Hare (Chicago).

[63] Barkshire I.R., Greenwood J.C., Kenny P.G., Prutton M., Roberts R.H. and El Gomati M.M., Surf. Interface Anal. 17 (1991) 209.

[64] Barkshire I.R., Prutton M., Greenwood J.C. and Prutton M., Appl. Surface Sci. 55 (1992) 245.

[65] El Gomati M.M., Prutton M., Lamb B. and Tuppen C. G., Surf. Interface Anal. 11 (1988) 251.

[66] Umbach A., Hoyer A., and Brunger R., Surf. Interface Anal. 14 (1989) 401.

[67] Barkshire I.R., Roberts R.H., Greenwood J.C., Kenny P.G., Prutton M. and El Gomati M.M., Inst. Phys. Conf. Ser. 119 (1991) 185.

[68] Ichimura S. and Shimizu R., Surf. Sci. 112 (1981) 386.

[69] Barkshire I.R., Prutton M. and Skinner D.K., Surf. Interface Anal. 17 (1991) 213.

[70] Moir P.A., Fitzgerald A.G. and Storey B.E., Surf. Interface Anal. 14 (1989) 295.

[71] Prutton M., Barkshire I.R. and Crone M., Ultramicroscopy (1995) in press.

[72] Crone M., Barkshire I.R. and Prutton M., Surf. Interface Anal. 21 (1994) 857.

[73] Walker C.G.H., Peacock D.C., Prutton M. and El Gomati M.M., Surf. Interface Anal. 11 (1988) 266.

[74] Abraham F.F. and Brundle C.R., J. Vac. Sci. Tech. 18 (1981) 506.

[75] Peacock D.C., Applied Surf. Sci. 26 (1986) 306.

[76] Peacock D.C., Applied Surf. Sci. 27 (1986) 58.

[77] Skinner D.K., Surf. Interface Anal. 14 (1989) 567.

[78] Tatlock G.J., Beahan P.G., Dare D., Hetherington C.J.D., Eaglesham D.J. and Kvam E.P., Inst. Phys. Conf. Ser. 90 (1987) 19.

[79] Prutton M., Walker C.G.H., Greenwood J.C., Kenny P.G., Dee J.C., Barkshire I.R., Roberts R.H. and El Gomati M.M., Surf. Interface Anal. 17 (1991) 71.

[80] Greenwood J.C., Lamb B. and Prutton M., Surf. Interface Anal. 20 (1992) 524.

[81] El Gomati M.M., Barkshire I.R., Greenwood J.C., Kenny P.G., Roberts R.H. and Prutton M., Microscopy: The Key Research Tool. 22 (1992) 29-38, (The Electron Microscopy Society of America, Milwaukee, WI, U.S.A.).

[82] Oechsner H., 1994 Le Vide, in press.

[83] Hösler W., Surf. Interface Anal. 17 (1991) 543.

[84] Barkshire I.R., Prutton M. and Smith G.C., Appl. Surf. Sci. 84 (1995) 331.

[85] Paparazzo E., Moretto L., D’Amato Cl. and Palmieri A., Appl. Surf. Sci. 74 (1994) 61.

[86] Plinius Secundus C. Naturalis Historia XXXI 58. 